Supporting Information for

\title{
Mimicking the Constrained Geometry of a Nitrogen-Fixation Intermediate
}

\author{
Tianchang Liu, Michael R. Gau, and Neil C. Tomson* \\ P. Roy and Diana T. Vagelos Laboratories, Department of Chemistry, \\ University of Pennsylvania, Philadelphia, Pennsylvania, 19104, United States
}

\section{Table of Contents}

1. Experimental Details and Characterization Data for All New Compounds .52

2. Summary of Crystallographic Data and Comparison to the Literature .........................................S6

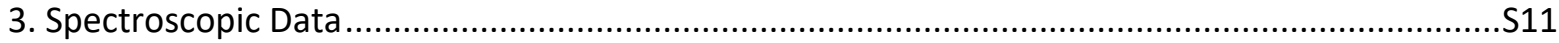

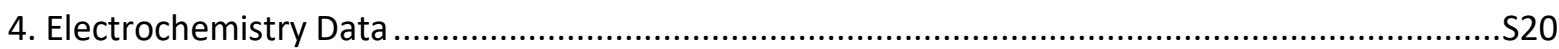

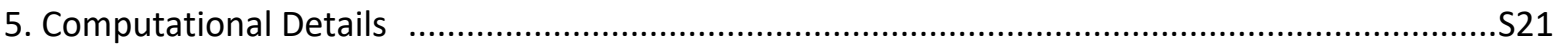

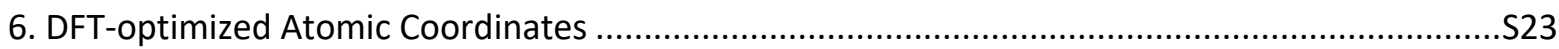

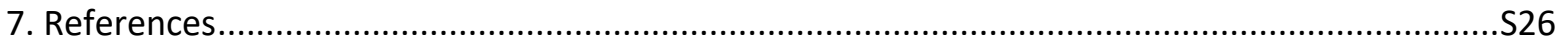




\section{Experimental Details and Characterization Data for All New Compounds}

General Methods. All reactions involving transition metals were performed under an inert atmosphere of $\mathrm{N}_{2}$ either in a PureLab HE glovebox or using standard Schlenk line technique. Glassware, stir bars, filter aid (Celite) and $4 \AA$ molecular sieves were dried in an oven at $150{ }^{\circ} \mathrm{C}$ for at least $12 \mathrm{~h}$ prior to use. All solvents ( $n$-pentane, $n$-hexane, diethyl ether, fluorobenzene, THF) were dried by passage through a column of activated alumina, deoxygenated by sparging with $\mathrm{N}_{2}$ for 15 min, and stored over $4 \AA$ molecular sieves. Deuterated solvents were purchased from Cambridge Isotope Laboratories, Inc., and dried over $\mathrm{Na}$ /benzophenone $\left(\mathrm{C}_{6} \mathrm{D}_{6}, \mathrm{THF}-d_{8}\right)$, distilled and stored under $\mathrm{N}_{2}$ over activated $4 \AA$ molecular sieves. ${ }^{15} \mathrm{~N}_{2}\left(98\right.$ atom $\left.\%{ }^{15} \mathrm{~N}\right)$ was purchased from MilliporeSigma and used without further purification. $\quad\left({ }^{3} \mathrm{PDI}_{2}\right) \operatorname{Sr}(\mathrm{OTf})_{2}{ }^{1}$ $\left[\left({ }^{3} \mathrm{PDI}_{2}\right) \mathrm{Fe}_{2}(\mu-\mathrm{Cl})\left(\mathrm{PPh}_{3}\right)_{2}\right][\mathrm{OTf}] \quad\left({ }^{\mathrm{Ph}}\left[\mathbf{F e}_{2} \mathbf{C l}\right]^{+}\right),{ }^{2} \quad\left[\left({ }^{3} \mathrm{PDI}_{2}\right) \mathrm{Fe}_{2}(\mu-\mathrm{Cl})\left(\mathrm{PMe}_{3}\right)_{2}\right][\mathrm{OTf}] \quad\left({ }^{\mathbf{M e}}\left[\mathbf{F e}_{2} \mathbf{C l}\right]^{+}\right)^{3}$ was synthesized according to literature procedures. $\mathrm{KC}_{8}$ was synthesized according to literature procedures and stored at $-35{ }^{\circ} \mathrm{C}$ in a glovebox prior to use. ${ }^{4} \mathrm{PPh}_{3}$ was purchased from MilliporeSigma, purified by recrystallization from hot ethanol and dried under vacuum $(30 \mathrm{mbar})$ at $40{ }^{\circ} \mathrm{C}$ for $6 \mathrm{~h}$ prior to use. ${ }^{5}$ $\mathrm{PMe}_{3}(98 \%)$ was either purchased from Strem Chemicals or synthesized according to literature procedures and stored as a $1 \mathrm{M}$ solution in THF at $-35{ }^{\circ} \mathrm{C}$ under dry nitrogen in a glovebox. ${ }^{6}$ 1,3,5-trimethoxybenzene was purchased from Alfa Aesar, recrystallized twice from $n$-hexane, followed by drying under vacuum $(30 \mathrm{mbar})$ at $60^{\circ} \mathrm{C}$ for $10 \mathrm{~h}$ prior to use. ${ }^{5}\left[{ }^{n} \mathrm{Bu}_{4} \mathrm{~N}\right]\left[\mathrm{PF}_{6}\right](98 \%)$ was purchased from MilliporeSigma and recrystallized twice from hot ethanol, followed by drying at 60 ${ }^{\circ} \mathrm{C}$ under 30 mbar for $10 \mathrm{~h}$ prior to use. ${ }^{5}$ Anhydrous $\mathrm{FeCl}_{2}$ was purchased from Strem Chemicals and used without further purification. ${ }^{1} \mathrm{H},{ }^{2} \mathrm{H},{ }^{13} \mathrm{C}\left\{{ }^{1} \mathrm{H}\right\},{ }^{31} \mathrm{P}\left\{{ }^{1} \mathrm{H}\right\},{ }^{15} \mathrm{~N},{ }^{1} \mathrm{H}-{ }^{13} \mathrm{C}$ HSQC and ${ }^{1} \mathrm{H}-{ }^{1} \mathrm{H}$ COSY NMR spectra were recorded on Bruker DMX 360, UNI 400, AV3BIO 500, UNI 500 or NEO 600 spectrometers. All chemical shifts $(\delta)$ are reported in units of ppm, with references to the residual protio-solvent resonance for proton and carbon chemical shifts. External $\mathrm{H}_{3} \mathrm{PO}_{4}$ and $\mathrm{CH}_{3} \mathrm{NO}_{2}$ were used for referencing ${ }^{31} \mathrm{P}$ and ${ }^{15} \mathrm{~N}$ NMR chemical shifts, respectively. Elemental analysis was performed by Midwest Microlab, LLC. IR samples were prepared as $\mathrm{KBr}$ pellets using powdered $\mathrm{KBr}$ that had been dried under vacuum at $130{ }^{\circ} \mathrm{C}$ for $4 \mathrm{~h}$. The water detected by IR spectroscopy as a wide band at $3440 \mathrm{~cm}^{-1}$ results from atmospheric hydration of the surface of the pellet in the 50-60 sec between removal of the sample from an inert atmosphere and the collection of IR data on a JASCO FT/IR-480 Plus spectrometer.

X-ray Crystallography. Single-crystal X-ray diffraction data were collected on a Rigaku XtaLAB Synergy-S HPC area detector (Dectris PILATUS3 R 200K) diffractometer with

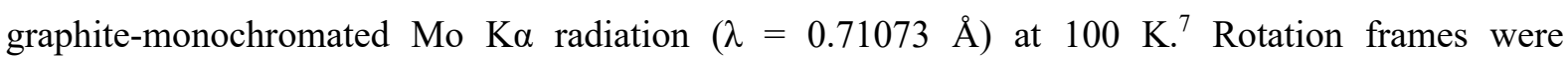
integrated using SAINT, ${ }^{8}$ producing a listing of unaveraged $\mathrm{F}^{2}$ and $\sigma\left(\mathrm{F}^{2}\right)$ values. The intensity data were corrected for Lorentz and polarization effects and for absorption using either SADABS $\left({ }^{\mathbf{M e}}\left[\mathbf{F e}_{2} \mathbf{N}_{2}\right]^{0}\right)$ or SCALE3 ABSPACK $\left({ }^{\mathbf{P h}}\left[\mathbf{F e}_{2} \mathbf{N}_{2}\right]^{0}\right) .{ }^{9}{ }^{10}$ The structures were solved by direct methods by 
using SHELXT and refined by full-matrix least squares, based on $\mathrm{F}^{2}$ using SHELXL-2018. Crystal parameters and refinement results are given in Table S1 and Table S2.

Computational Methods. Density functional theory (DFT) calculations were performed with the ORCA program package, v3.0.3. ${ }^{11}$ The geometry optimization was carried out at the B97-D3 level of DFT, ${ }^{12}$ using a model compound derived from crystallographic data, with the $p{ }^{-}{ }^{t} \mathrm{Bu}$ groups truncated to hydrogens. The def2-TZVP basis sets and the def2-TZVP/J auxiliary basis sets (used to expand the electron density in the resolution-of-identity (RI) approach) were used for Fe, P, and $\mathrm{N} .{ }^{13}$ All other atoms were described using the def2-SV(P) basis sets and def2-SV/J auxiliary basis sets. The SCF calculations were tightly converged $\left(1 \times 10^{-8} \mathrm{E}_{\mathrm{h}}\right.$ in energy, $1 \times 10^{-7} \mathrm{E}_{\mathrm{h}}$ in the density change, and $5 \times 10^{-7}$ in the maximum element of the DIIS error vector). The geometry was considered converged after the energy change was less than $1 \times 10^{-6} \mathrm{E}_{\mathrm{h}}$, the gradient norm and maximum gradient element were smaller than $3 \times 10^{-4} \mathrm{E}_{\mathrm{h}}-\mathrm{Bohr}^{-1}$ and $1 \times 10^{-4} \mathrm{E}_{\mathrm{h}}-\mathrm{Bohr}^{-1}$, respectively, and the root-mean square and maximum displacements of all atoms were smaller than $6 \times 10^{-4}$ Bohr and $1 \times 10^{-3}$ Bohr, respectively. A numerical frequency calculation was used to verify that the calculated structure represented a local minimum on the potential energy surface. The reported energy is a Gibbs free energy, calculated for $298.15 \mathrm{~K}$ and $1.00 \mathrm{~atm}$, as obtained from the numerical frequency calculation on the optimized geometry. Plots were generated using the program Chimera, ${ }^{14}$ with isosurface cutoffs of $|0.025|$ a.u.

Electrochemistry. Cyclic voltammetry experiments were performed using a BASi C3 Cell Stand paired with an Epsilon E2 Potentiostat. The data were processed with BASi Epsilon-EC software version 2.13.77. All experiments were performed under an $\mathrm{N}_{2}$ atmosphere in a VAC OMNI-LAB glovebox using an electrochemical cell that consists of a glassy carbon ( $3 \mathrm{~mm}$ outer diameter) working electrode, a platinum wire counter electrode and a $\mathrm{AgPF}_{6}(100 \mathrm{mM}$ in THF $) / \mathrm{Ag}$ reference electrode. All experiments were conducted in THF, with $1 \mathrm{mM}$ analyte and $100 \mathrm{mM}\left[{ }^{n} \mathrm{Bu}_{4} \mathrm{~N}\right]\left[\mathrm{PF}_{6}\right]$ as the supporting electrolyte. Potentials were reported versus $\mathrm{Cp}_{2} \mathrm{Fe}^{+/ 0}$, which was added as an internal standard for reference at the end of each experiment.

Synthesis of $\left({ }^{3} \mathbf{P D I}_{2}\right) \mathbf{F e}_{2}\left(\boldsymbol{\mu}-\mathbf{N}_{2}\right)\left(\mathbf{P P h}_{3}\right)_{2}\left({ }^{\mathbf{P h}}\left[\mathbf{F e}_{2} \mathbf{N}_{2}\right]^{0}\right)$. A KC 8 (28.9 mg, $\left.0.22 \mathrm{mmol}\right)$ suspension in THF was chilled to $-35{ }^{\circ} \mathrm{C}$, then added dropwise to a pre-chilled, stirred solution of ${ }^{\mathrm{Ph}}\left[\mathrm{Fe}_{2} \mathbf{C l}\right]^{+}(144 \mathrm{mg}$, $0.11 \mathrm{mmol}$ ) in $10 \mathrm{~mL}$ THF, resulting in a rapid color change from dark brown to purplish red. The mixture was allowed to warm to room temperature and stirred for 1 hour. All volatile materials were then removed under vacuum. The residue was treated with $5 \mathrm{~mL} \mathrm{Et}_{2} \mathrm{O}$, then filtered through Celite and stored at $-35{ }^{\circ} \mathrm{C}$ for three days to afford ${ }^{\mathrm{Ph}}\left[\mathbf{F e}_{2} \mathbf{N}_{2}\right]^{0}$ as dark red needles. Yield $48.0 \mathrm{mg}(35 \%)$. Analytically pure material was obtained following recrystallization under the same conditions. ${ }^{1} \mathrm{H}$ NMR (600 MHz, THF- $\left.d_{8}, 298 \mathrm{~K}\right): \delta=7.69(\mathrm{~s}, 4 \mathrm{H}, \mathrm{py} m-H), 7.16\left(\mathrm{t},{ }^{3} J_{\mathrm{HH}}=9.3 \mathrm{~Hz}, 6 \mathrm{H}, \operatorname{Ar} H\right), 7.07$ (t, $\left.{ }^{3} J_{\mathrm{HH}}=8.4 \mathrm{~Hz}, 12 \mathrm{H}, \operatorname{Ar} H\right), 6.79\left(\mathrm{t},{ }^{3} J_{\mathrm{HH}}=9.3 \mathrm{~Hz}, 12 \mathrm{H}, \operatorname{Ar} H\right), 4.39-4.34\left(\mathrm{~m}, 4 \mathrm{H}, \mathrm{CH}_{2}\right), 3.86-3.82$ $\left(\mathrm{m}, 4 \mathrm{H}, \mathrm{CH}_{2}\right), 2.46-2.38\left(\mathrm{~m}, 2 \mathrm{H}, \mathrm{CH}_{2}\right), 2.13\left(\mathrm{~d},{ }^{5} \mathrm{~J}_{\mathrm{PH}}=5.4 \mathrm{~Hz}, 12 \mathrm{H}, \mathrm{CH}_{3}\right), 1.35\left(\mathrm{~s}, 18 \mathrm{H}, \mathrm{C}\left(\mathrm{CH}_{3}\right)_{3}\right)$, $0.32-0.27\left(\mathrm{~m}, 2 \mathrm{H}, \mathrm{C} H_{2}\right) \mathrm{ppm} ;{ }^{13} \mathrm{C}\left\{{ }^{1} \mathrm{H}\right\} \mathrm{NMR}\left(600 \mathrm{MHz}, \mathrm{THF}-d_{8}, 298 \mathrm{~K}\right): \delta=145.88\left(\mathrm{~d},{ }^{3} J_{\mathrm{PC}}=15.6\right.$ $\mathrm{Hz}, \operatorname{ArC}), 141.83\left(\mathrm{~d},{ }^{3} J_{\mathrm{PC}}=21 \mathrm{~Hz}, C_{\text {imine }}\right), 137.75(\mathrm{~s}, \operatorname{ArC}), 109.76(\mathrm{~s}, \operatorname{ArC}), 61.10\left(\mathrm{~s}, C_{\mathrm{H}}\right), 42.04$ (t, 
$\left.{ }^{4} J_{\mathrm{PC}}=30 \mathrm{~Hz}, C \mathrm{H}_{2}\right), 35.68\left(\mathrm{~s}, C\left(\mathrm{CH}_{3}\right)_{3}\right), 32.39\left(\mathrm{~s}, \mathrm{C}\left(\mathrm{CH}_{3}\right)_{3}\right), 15.90\left(\mathrm{~d},{ }^{2} J_{\mathrm{PC}}=68.4 \mathrm{~Hz}, \mathrm{P}\left(\mathrm{CH}_{3}\right)_{3}\right), 13.93$

$\left(\mathrm{d},{ }^{2} J_{\mathrm{PC}}=11.4 \mathrm{~Hz}, C \mathrm{H}_{3}\right) \mathrm{ppm} ;{ }^{31} \mathrm{P}\left\{{ }^{1} \mathrm{H}\right\} \mathrm{NMR}\left(500 \mathrm{MHz}, \mathrm{THF}-d_{8}, 298 \mathrm{~K}\right): \delta=60.97\left(\mathrm{~s}, P\left(\mathrm{CH}_{3}\right)_{3}\right) \mathrm{ppm}$. FT-IR (KBr pellet): $v_{\mathrm{N}-\mathrm{N}}=1959 \mathrm{~cm}^{-1}$. Anal. Calcd. for $\mathrm{C}_{68} \mathrm{H}_{76} \mathrm{Fe}_{2} \mathrm{~N}_{8} \mathrm{P}_{2} \cdot 2 \mathrm{C}_{4} \mathrm{H}_{8} \mathrm{O}(1327.27 \mathrm{~g} / \mathrm{mol})$ : C, 68.77; H, 7.29; N, 8.44. Found: C, 68.14; H, 7.00; N, 8.77.

Alternative synthesis of $\left({ }^{3} \mathrm{PDI}_{2}\right) \mathrm{Fe}_{2}\left(\mu-\mathrm{N}_{2}\right)\left(\mathrm{PPh}_{3}\right)_{2} \quad\left({ }^{\mathrm{Ph}}\left[\mathrm{Fe}_{2} \mathbf{N}_{2}\right]^{0}\right)$. To a stirred solution of $\left({ }^{3} \mathrm{PDI}_{2}\right) \operatorname{Sr}(\mathrm{OTf})_{2}(200 \mathrm{mg}, 0.222 \mathrm{mmol})$ in THF $(7 \mathrm{~mL})$ was added solid, anhydrous $\mathrm{FeCl}_{2}(56.4 \mathrm{mg}$, $0.444 \mathrm{mmol}$ ) at room temperature. After being stirred for $2 \mathrm{~h}$, the purple slurry was treated with $\mathrm{PPh}_{3}$ (116.4 mg, 0. $444 \mathrm{mmol}$ ). The reaction mixture was stirred for an additional $10 \mathrm{~min}$, then chilled to $-35{ }^{\circ} \mathrm{C}$. A suspension of $\mathrm{KC}_{8}(60 \mathrm{mg}, 0.444 \mathrm{mmol})$ in THF $(2 \mathrm{~mL})$ was chilled to $-35{ }^{\circ} \mathrm{C}$ and added into the reaction mixture. The resulting dark green slurry was allowed to warm to room temperature and stirred for $1 \mathrm{~h}$, during which time the color gradually turned dark brown. The reaction mixture was filtered through Celite, then chilled again to $-35{ }^{\circ} \mathrm{C}$. A second portion of $\mathrm{KC}_{8}(60 \mathrm{mg}, 0.444$ mmol) in THF ( $2 \mathrm{~mL}$ ) was chilled to $-35^{\circ} \mathrm{C}$ and added into the reaction mixture. The reaction mixture was allowed to warm to room temperature and stirred for $1 \mathrm{~h}$, during which time the slurry gradually turned purplish red. The volatile materials were removed under reduced pressure. The solid residue was extracted with $7 \mathrm{~mL}$ of diethyl ether, filtered through Celite, concentrated to $3 \mathrm{~mL}$, filtered again through Celite and stored at $-35{ }^{\circ} \mathrm{C}$ for $3 \mathrm{~d}$ to afford ${ }^{\mathrm{Ph}}\left[\mathbf{F e}_{2} \mathbf{N}_{2}\right]^{\mathbf{0}}$ as dark red needles. Yield $44 \mathrm{mg}$ (17.4 $\%$ ). The material obtained by this method displayed the same analytical data as those given above.

Synthesis of $\left[\left({ }^{3} \mathrm{PDI}_{2}\right) \mathrm{Fe}_{2}\left(\boldsymbol{\mu l}^{-15} \mathbf{N}_{2}\right)\left(\mathrm{PPh}_{3}\right)_{2}\right]\left({ }^{\mathrm{Ph}}\left[\mathrm{Fe}_{2}{ }^{15} \mathbf{N}_{2}\right]^{0}\right)$. The synthesis of ${ }^{\mathrm{Ph}}\left[\mathrm{Fe}_{2}{ }^{15} \mathbf{N}_{2}\right]^{0}$ was prepared according to the general procedure given above for the alternative synthesis of ${ }^{\mathrm{Ph}}\left[\mathrm{Fe}_{2} \mathbf{N}_{2}\right]^{0}$, but with the following modifications. The dark brown slurry obtained following the initial addition of $\mathrm{KC}_{8}$ was added to a $100 \mathrm{~mL}$ Schlenk flask. The flask was cooled using a dry ice/acetone both, then evacuated for $c a .1 \mathrm{~min}$ and refilled with Ar; this procedure was repeated two times. The flask was then charged with a sealed glass capsule containing the second portion of $\mathrm{KC}_{8}$. After resealing the flask and evacuating it for $1 \mathrm{~min}$, the headspace was refilled with $c a .1$ atm of ${ }^{15} \mathrm{~N}_{2}$. The glass capsule was broken with vigorous stirring, then the flask was allowed to warm to $0{ }^{\circ} \mathrm{C}$ and stirred for $30 \mathrm{~min}$. The color of the solution turned from brown to red on warming. The mixture was then allowed to obtain room temperature and stirred for $1 \mathrm{~h}$ before workup, as described above. Yield $40.7 \mathrm{mg}$ (15.5\% on $0.222 \mathrm{mmol} \mathrm{scale}) .{ }^{15} \mathrm{~N}$ NMR $\left(61 \mathrm{MHz}\right.$, THF- $\left.d_{8}, 25{ }^{\circ} \mathrm{C}\right): \delta=355.2\left(\mathrm{~s}, \mathrm{Fe}_{2}{ }^{15} N_{2}\right) \mathrm{ppm}$. The product was determined to be $93 \%$ pure by comparison with an internal standard of 1,3,5-trimethoxybenzene. FT-IR (KBr pellet): $v_{15 \mathrm{~N}-15 \mathrm{~N}}=1896 \mathrm{~cm}^{-1}$.

Synthesis of $\left({ }^{3} \mathrm{PDI}_{2}\right) \mathrm{Fe}_{2}\left(\boldsymbol{\mu}-\mathbf{N}_{2}\right)\left(\mathrm{PMe}_{3}\right)_{2}\left({ }^{\mathrm{Me}}\left[\mathrm{Fe}_{2} \mathbf{N}_{2}\right]^{0}\right)$. A solution of ${ }^{\mathrm{Me}}\left[\mathrm{Fe}_{2} \mathbf{C l}\right]^{+}(109 \mathrm{mg}, 0.08 \mathrm{mmol})$ in THF $(10 \mathrm{~mL})$ was chilled to $-35^{\circ} \mathrm{C}$. A slurry of $\mathrm{KC}_{8}(30 \mathrm{mg}, 0.222 \mathrm{mmol})$ in THF $(2 \mathrm{~mL})$ was also chilled to $-35{ }^{\circ} \mathrm{C}$, then slowly added the reaction mixture, resulting in a rapid color change from dark brown to red. The mixture was allowed to warm to room temperature and stirred for 1 hour. All volatile materials were then removed under reduced pressure. The reaction mixture was then extracted with $5 \mathrm{~mL} \mathrm{Et} t_{2} \mathrm{O}$, filtered through Celite and stored at $-35{ }^{\circ} \mathrm{C}$ for three days to afford 
${ }^{\mathrm{Me}}\left[\mathrm{Fe}_{2} \mathbf{N}_{2}\right]^{0}$ as dark red needles. Yield $10.1 \mathrm{mg}$ (11\%). Following recrystallization under the conditions described above, spectroscopically pure material was obtained, as determined by ${ }^{1} \mathrm{H}$ NMR spectroscopy in the presence of an internal standard. ${ }^{1} \mathrm{H}$ NMR (600 MHz, THF- $\left.d_{8}, 298 \mathrm{~K}\right): \delta=7.77$ (s, 4H, py $m-H), 5.09-5.05\left(\mathrm{~m}, 4 \mathrm{H}, \mathrm{CH}_{2}\right), 4.53-4.45(\mathrm{~m}, 4 \mathrm{H}, \mathrm{CH}), 2.66-2.62\left(\mathrm{~m}, 2 \mathrm{H}, \mathrm{CH}_{2}\right), 2.39$ $\left(\mathrm{d},{ }^{5} \mathrm{~J}_{\mathrm{PH}}=5.4 \mathrm{~Hz}, 12 \mathrm{H}, \mathrm{CH}\right), 1.38\left(\mathrm{~s}, 18 \mathrm{H}, \mathrm{C}\left(\mathrm{CH}_{3}\right)_{3}\right), 0.59\left(\mathrm{~d},{ }^{2} J_{\mathrm{PH}}=7.2 \mathrm{~Hz}, 18 \mathrm{H}, \mathrm{P}\left(\mathrm{CH}_{3}\right)_{3}\right), 0.46-$ $0.41(\mathrm{~m}, 2 \mathrm{H}, \mathrm{CH}) \mathrm{ppm} ;{ }^{13} \mathrm{C}\left\{{ }^{1} \mathrm{H}\right\}$ NMR $\left(600 \mathrm{MHz}, \mathrm{THF}-d_{8}, 298 \mathrm{~K}\right): \quad \delta=145.88\left(\mathrm{~d},{ }^{3} J_{\mathrm{PC}}=15.6 \mathrm{~Hz}\right.$, $\operatorname{ArC}), 141.83\left(\mathrm{~d},{ }^{3} J_{\mathrm{PC}}=21 \mathrm{~Hz}, C_{\text {imine }}\right), 137.75(\mathrm{~s}, \operatorname{ArC}), 109.76(\mathrm{~s}, \operatorname{ArC}), 61.10\left(\mathrm{~s}, C \mathrm{H}_{2}\right), 42.04\left(\mathrm{t},{ }^{4} J_{\mathrm{PC}}\right.$ $\left.=30 \mathrm{~Hz}, \mathrm{CH}_{2}\right), 35.68\left(\mathrm{~s}, \mathrm{C}\left(\mathrm{CH}_{3}\right)_{3}\right), 32.39\left(\mathrm{~s}, \mathrm{C}\left(\mathrm{CH}_{3}\right)_{3}\right), 15.90\left(\mathrm{~d},{ }^{2} J_{\mathrm{PC}}=68.4 \mathrm{~Hz}, \mathrm{P}\left(\mathrm{CH}_{3}\right)_{3}\right), 13.93(\mathrm{~d}$, $\left.{ }^{2} J_{\mathrm{PC}}=11.4 \mathrm{~Hz}, C \mathrm{H}_{3}\right) \mathrm{ppm} ;{ }^{31} \mathrm{P}\left\{{ }^{1} \mathrm{H}\right\} \mathrm{NMR}\left(500 \mathrm{MHz}, \mathrm{THF}-d_{8}, 298 \mathrm{~K}\right): \delta=22.33\left(\mathrm{~s}, P\left(\mathrm{CH}_{3}\right)_{3}\right) \mathrm{ppm}$. FT-IR ( $\mathrm{KBr}$ pellet): $v_{\mathrm{N}-\mathrm{N}}=2003 \mathrm{~cm}^{-1}$. ${ }^{\mathbf{M e}}\left[\mathbf{F e}_{2} \mathbf{N}_{2}\right]^{0}$ was observed to undergo slow thermal decomposition, which prevented the collection of suitable microanalytical data. 


\section{Summary of Crystallographic Data}

Table S1. Summary of crystallographic data for compounds reported in this work.

\begin{tabular}{|c|c|c|}
\hline Complexes & ${ }^{\mathrm{Ph}}\left[\mathrm{Fe}_{2} \mathrm{~N}_{2}\right]^{0} \cdot \mathbf{2 E t _ { 2 } \mathrm { O }}$ & ${ }^{\mathrm{Me}}\left[\mathrm{Fe}_{2} \mathrm{~N}_{2}\right]^{0} \cdot \mathrm{Et}_{2} \mathrm{O} \cdot{ }^{1} /{ }_{2}$ Pentane \\
\hline Empirical formula & $\mathrm{C}_{76} \mathrm{H}_{96} \mathrm{Fe}_{2} \mathrm{~N}_{8} \mathrm{O}_{2} \mathrm{P}_{2}$ & $\mathrm{C}_{45} \mathrm{H}_{81} \mathrm{Fe}_{2} \mathrm{~N}_{8} \mathrm{OP}_{2}$ \\
\hline Formula weight & 1327.27 & 923.81 \\
\hline Temperature/K & 100 & 100 \\
\hline Crystal system & triclinic & triclinic \\
\hline Space group & P-1 & $\mathrm{P}-1$ \\
\hline $\mathrm{a} / \AA$ & $10.6831(2)$ & $9.8631(6)$ \\
\hline $\mathrm{b} / \AA$ & $16.4143(4)$ & $13.0959(8)$ \\
\hline $\mathrm{c} / \AA \AA$ & $20.2856(4)$ & $20.1607(12)$ \\
\hline$\alpha /^{\circ}$ & $91.674(2)$ & $91.874(2)$ \\
\hline$\beta /{ }^{\circ}$ & $98.0110(10)$ & $92.340(2)$ \\
\hline$\gamma /{ }^{\circ}$ & $98.486(2)$ & $106.816(2)$ \\
\hline Volume $/ \AA^{3}$ & $3479.41(13)$ & $2487.9(3)$ \\
\hline $\mathrm{Z}$ & 2 & 2 \\
\hline$\rho_{\text {cald }} / \mathrm{g} \cdot \mathrm{cm}^{-3}$ & 1.267 & 1.233 \\
\hline$\mu / \mathrm{mm}^{-1}$ & 0.515 & 0.688 \\
\hline $\mathrm{F}(000)$ & 1412.0 & 994.0 \\
\hline Crystal size $/ \mathrm{mm}^{3}$ & $0.34 \times 0.12 \times 0.04$ & $0.27 \times 0.23 \times 0.17$ \\
\hline Radiation & $\operatorname{Mo~K}_{\alpha}(\lambda=0.71073)$ & $\operatorname{Mo~K}_{\alpha}(\lambda=0.71073)$ \\
\hline $2 \theta$ range for data collection & $4.06-55.142^{\circ}$ & 5.048 to $55.096^{\circ}$ \\
\hline Index ranges & $\begin{array}{l}-13 \leq \mathrm{h} \leq 13 \\
-21 \leq \mathrm{k} \leq 21 \\
-26 \leq 1 \leq 26\end{array}$ & $\begin{array}{l}-12 \leq \mathrm{h} \leq 12 \\
-16 \leq \mathrm{k} \leq 17 \\
-26 \leq 1 \leq 26\end{array}$ \\
\hline Reflections collected & 127175 & 52187 \\
\hline Independent reflections & $26672\left[\mathrm{R}_{\mathrm{int}}=0.070\right]$ & $11406\left[\mathrm{R}_{\text {int }}=0.0413\right]$ \\
\hline Data/restraints/parameters & $26672 / 0 / 826$ & $11406 / 0 / 542$ \\
\hline Goodness-of-fit on $\mathrm{F}^{2}$ & 1.029 & 1.111 \\
\hline Final $R$ indexes $[I>=2 \sigma(I)]$ & $\mathrm{R}_{1}=0.0557, \mathrm{wR}_{2}=0.1431$ & $\mathrm{R}_{1}=0.0440, \mathrm{wR}_{2}=0.1000$ \\
\hline Final $\mathrm{R}$ indexes [all data] & $\mathrm{R}_{1}=0.0672, \mathrm{wR}_{2}=0.1501$ & $\mathrm{R}_{1}=0.0603, \mathrm{wR}_{2}=0.1063$ \\
\hline Largest diff. peak/hole / e $\bullet \AA^{-3}$ & $0.87 /-0.47$ & $0.71 /-0.53$ \\
\hline
\end{tabular}


Table S2. Selected bond lengths $(\AA)$ and angles $\left(^{\circ}\right)$ for experimental and DFT computational data.

\begin{tabular}{|c|c|c|c|}
\hline & $\begin{array}{c}{ }^{\mathrm{Ph}}\left[\mathrm{Fe}_{2} \mathbf{N}_{2}\right]^{\mathbf{0}} \\
(\mathrm{XRD})\end{array}$ & $\begin{array}{c}{ }^{\mathrm{Me}}\left[\mathbf{F e}_{2} \mathbf{N}_{2}\right]^{\mathbf{0}} \\
(\mathrm{XRD})\end{array}$ & $\begin{array}{c}{ }^{\mathrm{Me}}\left[\mathbf{F e}_{2} \mathbf{N}_{2}\right]^{\mathbf{0}}{ }^{(\mathrm{DFT})}\end{array}$ \\
\hline $\mathrm{Fe}-\mathrm{Fe}$ & $4.5628(6)$ & $4.6066(6)$ & 4.536 \\
\hline \multirow[t]{2}{*}{$\mathrm{Fe}-\mathrm{N}_{\mathrm{N} 2}$} & $1.835(3)$ & $1.828(2)$ & 1.802 \\
\hline & $1.823(3)$ & $1.811(2)$ & 1.802 \\
\hline \multirow[t]{4}{*}{$\mathbf{F e}-\mathbf{N}_{\text {imine }}$} & $1.918(3)$ & $1.902(2)$ & 1.962 \\
\hline & $1.924(3)$ & $1.910(2)$ & 1.962 \\
\hline & $1.921(3)$ & $1.911(2)$ & 1.963 \\
\hline & $1.926(3)$ & $1.909(2)$ & 1.964 \\
\hline \multirow[t]{2}{*}{$\mathbf{F e}-\mathbf{N}_{\mathrm{py}}$} & $1.830(3)$ & $1.814(2)$ & 1.857 \\
\hline & $1.831(3)$ & $1.822(2)$ & 1.858 \\
\hline $\mathbf{N}_{\mu}-\mathbf{N}_{\mu}$ & $1.139(3)$ & $1.135(3)$ & 1.148 \\
\hline \multirow[t]{4}{*}{$N_{\text {imine }}-C_{\text {imine }}$} & $1.333(4)$ & $1.345(3)$ & 1.350 \\
\hline & $1.338(4)$ & $1.339(3)$ & 1.350 \\
\hline & $1.338(4)$ & $1.343(3)$ & 1.350 \\
\hline & $1.339(4)$ & $1.340(3)$ & 1.350 \\
\hline \multirow[t]{4}{*}{$\mathbf{C}_{\text {imine }}-\mathbf{C}_{\mathbf{p y}}$} & $1.420(4)$ & $1.426(3)$ & 1.437 \\
\hline & $1.428(4)$ & $1.424(3)$ & 1.437 \\
\hline & $1.429(4)$ & $1.422(3)$ & 1.438 \\
\hline & $1.428(4)$ & $1.430(3)$ & 1.438 \\
\hline \multirow[t]{2}{*}{$\angle \mathrm{Fe}-\mathrm{N}_{\mu}-\mathrm{N}_{\mu}$} & $159.8(3)$ & $157.99(19)$ & 160.0 \\
\hline & $163.2(3)$ & $162.88(19)$ & 160.1 \\
\hline$\Delta$ & 0.067 & 0.061 & 0.064 \\
\hline
\end{tabular}




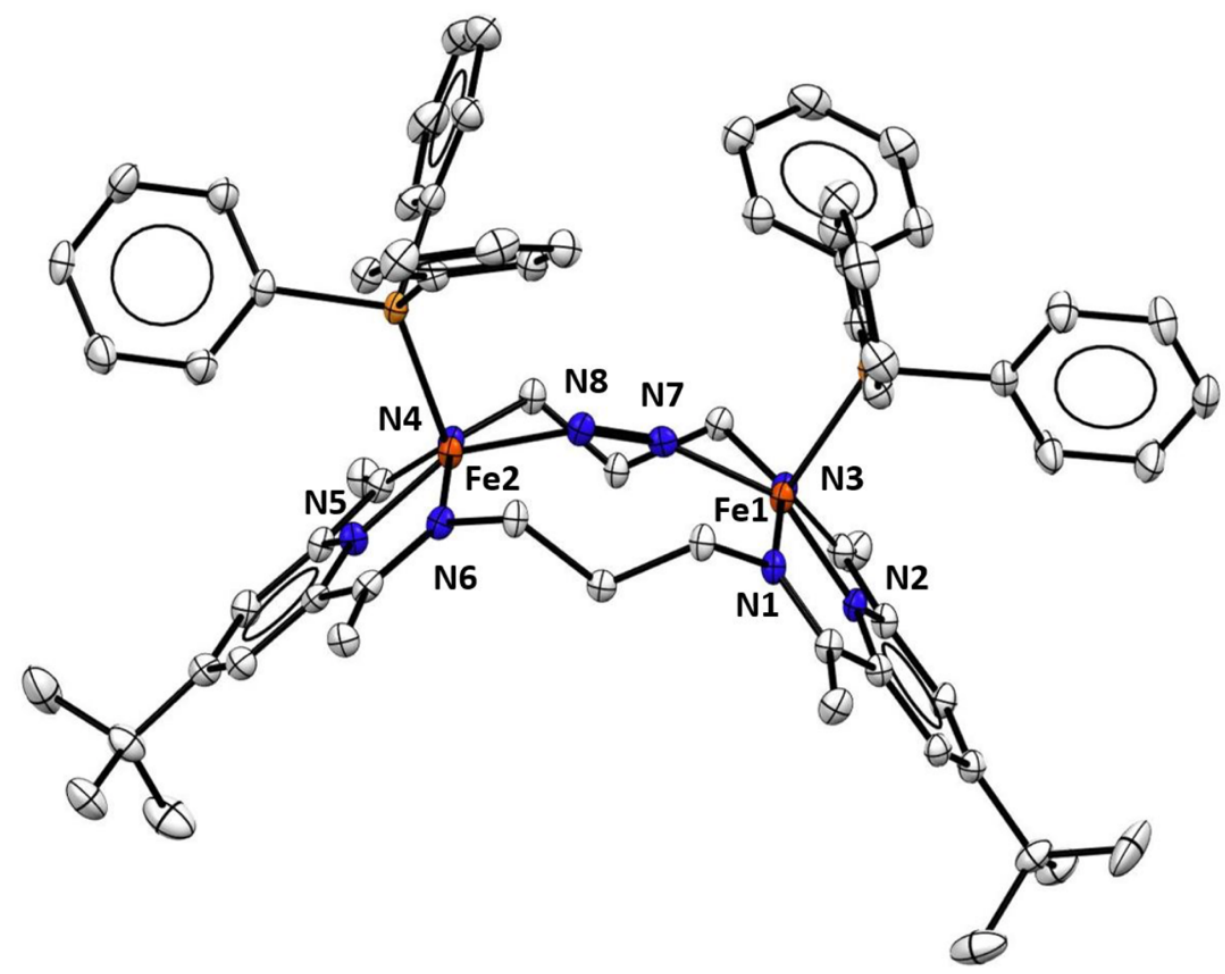

Figure S1. Crystal structure of ${ }^{\mathbf{P h}}\left[\mathbf{F e}_{2} \mathbf{N}_{2}\right]^{0}$, with thermal ellipsoids at the $50 \%$ probability level. The co-crystallized solvent molecules and hydrogen atoms were omitted for clarity.

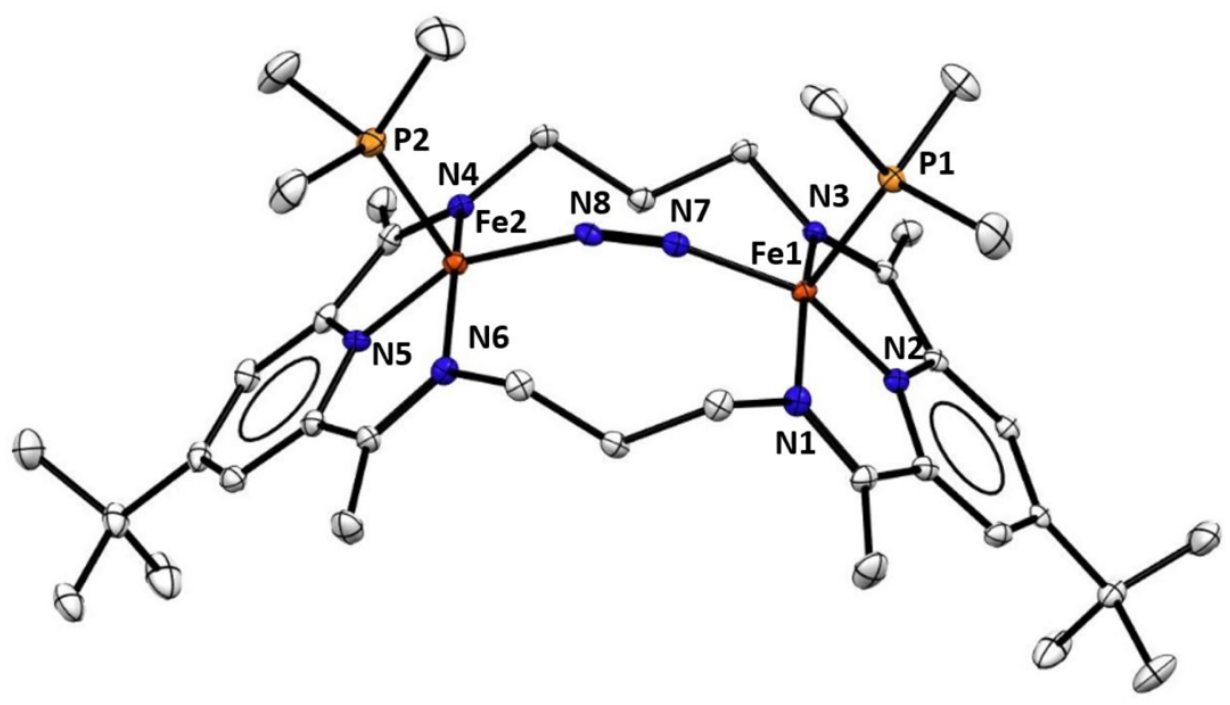

Figure S2. Crystal structure of ${ }^{\mathrm{Me}}\left[\mathbf{F e}_{2} \mathbf{N}_{2}\right]^{0}$, with thermal ellipsoids at the $50 \%$ probability level. The co-crystallized solvent molecules and hydrogen atoms were omitted for clarity. 
Table S3. Dinitrogen N-N bond lengths and stretching frequencies of neutral, di-iron bridging dinitrogen compounds (top) and other species with non-linear $\mathrm{M}-\mathrm{N}_{2}-\mathrm{M}$ bridges (bottom).

\begin{tabular}{|c|c|c|c|}
\hline complex & $\mathrm{N}-\mathrm{N}(\AA)$ & $v_{\mathrm{NN}}\left(\mathrm{cm}^{-1}\right)$ & ref \\
\hline$[\mathrm{Fe}(\operatorname{carb}-\mathrm{PNP})]_{2}\left(\mu-\mathrm{N}_{2}\right)$ & $1.106(4)$ & & 16 \\
\hline$\left[\left(\eta^{6}-\mathrm{C}_{6} \mathrm{H}_{6}\right) \mathrm{Fe}\left(\mathrm{Me}_{2} \mathrm{SiC}_{6} \mathrm{H}_{4} \mathrm{SiMe}_{2}\right)\right]_{2}\left(\mu-\mathrm{N}_{2}\right)$ & $1.119(3)$ & $2035^{b}$ & 17 \\
\hline$\left\{\left[\mathrm{PhBP}^{\mathrm{CH} 2 \mathrm{Cy}}{ }_{3}\right] \mathrm{Fe}(\mathrm{OAc})\right\}_{2}\left(\mu-\mathrm{N}_{2}\right)$ & $1.120(5)$ & $2083^{a}$ & 18 \\
\hline$\left[\{\mathrm{Fe}(\mathrm{N}, \text { arene-Piso })\}_{2}\left(\mu-\mathrm{N}_{2}\right)\right]$ & $1.124(6)$ & $2005^{b}$ & 19 \\
\hline$\left[\left(\eta^{6}-\mathrm{C}_{6} \mathrm{H}_{5} \mathrm{Me}\right) \mathrm{Fe}\left(\mathrm{Me}_{2} \mathrm{SiC}_{6} \mathrm{H}_{4} \mathrm{SiMe}_{2}\right)\right]_{2}\left(\mu-\mathrm{N}_{2}\right)$ & $1.126(3)$ & $2022^{b}$ & 17 \\
\hline$\left[\left(\mathrm{FeH}\left(\mathrm{PP}_{3}\right)\right)\left(\mu-\mathrm{N}_{2}\right)\left(\mathrm{Fe}\left(\mathrm{PP}_{3}\right)\right)\right]\left[\mathrm{BPh}_{4}\right]$ & $1.127(2)$ & & 20 \\
\hline$\left[\mathrm{Cp}^{*}\left(\mathrm{Ph}_{2} \mathrm{PC}_{6} \mathrm{H}_{4} \mathrm{~S}\right) \mathrm{Fe}\right]_{2}\left(\mu-\mathrm{N}_{2}\right)$ & $1.130(6)$ & $2016^{a}$ & 21 \\
\hline$\left[\mathrm{Fe}(\mathrm{CO})_{2}\left(\mathrm{P}\left(\mathrm{OCH}_{3}\right)_{3}\right)_{2}\right]\left(\mu-\mathrm{N}_{2}\right)$ & 1.13 & & 22 \\
\hline$[\mathrm{Fe}(\mathrm{PNNP}-\mathrm{Cy})]_{2}\left(\mu-\mathrm{N}_{2}\right)$ & $1.134(6)$ & & 23 \\
\hline$\left[\mathrm{Fe}(\mathrm{CO})_{2}\left(\mathrm{PEt}_{3}\right)_{2}\right]\left(\mu-\mathrm{N}_{2}\right)$ & $1.134(21)$ & & 24 \\
\hline${ }^{\mathrm{Me}}\left[\mathrm{Fe}_{2} \mathbf{N}_{2}\right]^{0}$ & $1.135(3)$ & $2003^{a}$ & this work \\
\hline$[\mathrm{Fe}(\mathrm{PNNP}-\mathrm{Ph})]_{2}\left(\mu-\mathrm{N}_{2}\right)$ & $1.135(4)$ & & 23 \\
\hline$\left\{\left[\mathrm{PhBP}^{\mathrm{iPr}}{ }_{3}\right] \mathrm{Fe}\right\}_{2}\left(\mu-\mathrm{N}_{2}\right)$ & $1.138(6)$ & & 25 \\
\hline$\left[\left(\mathrm{SiP}_{2} \mathrm{~S}\right) \mathrm{Fe}\right]_{2}\left(\mu-\mathrm{N}_{2}\right)$ & $1.138(2)$ & $1888^{a}$ & 26 \\
\hline${ }^{\mathrm{Ph}}\left[\mathrm{Fe}_{2} \mathbf{N}_{2}\right]^{0}$ & $1.139(3)$ & $1959^{a}$ & this work \\
\hline$\left[\left(\eta^{6}-\mathrm{C}_{6} \mathrm{H}_{3} \mathrm{Me}_{3}\right) \mathrm{Fe}\left(\mathrm{Me}_{2} \mathrm{SiC}_{6} \mathrm{H}_{4} \mathrm{SiMe}_{2}\right)\right]_{2}\left(\mu-\mathrm{N}_{2}\right)$ & 1.139 & $2012^{b}$ & 27 \\
\hline$\left[\left({ }^{\mathrm{Me}} \mathrm{CNC}\right) \mathrm{Fe}\left(\mathrm{N}_{2}\right)\right]_{2}\left(\mu-\mathrm{N}_{2}\right)$ & 1.140 & & 28 \\
\hline$\left[\mathrm{Fe}(\mathrm{dmpe})_{2}\right]_{2}\left(\mu-\mathrm{N}_{2}\right)$ & $1.144(3)$ & $1933^{b}$ & 29 \\
\hline$[\mathrm{Fe}(\mathrm{AltraPhos})]_{2}\left(\mu-\mathrm{N}_{2}\right)$ & $1.146(7)$ & $2010^{a}$ & 30 \\
\hline$\left[\mathrm{P}_{2}{ }^{\mathrm{P} P h} \mathrm{Fe}(\mathrm{H})\right]_{2}\left(\mu-\mathrm{N}_{2}\right)$ & 1.15 & & 31 \\
\hline$\left(\left[\mathrm{N}_{2} \mathrm{P}_{2}\right] \mathrm{Fe}\right)_{2}\left(\mu-\mathrm{N}_{2}\right)$ & $1.166(3)$ & $1760^{a}$ & 32 \\
\hline$\left[\left\{\mathrm{Fe}\left(\mathrm{Me}-\mathrm{PNP} \mathrm{p}^{\alpha \mathrm{H}}\right)\right\}_{2}\left(\mu-\mathrm{N}_{2}\right)\right]\left[\left(\mathrm{BAr}_{4}\right)_{2}\right]$ & $1.170(5)$ & & 33 \\
\hline$\left.\left[{ }^{i \mathrm{Pr}} \mathrm{DPB}\right) \mathrm{Fe}\right]_{2}\left(\mu-\mathrm{N}_{2}\right)$ & $1.170(5)$ & & 34 \\
\hline $\mathrm{Fe}(\text { depe })_{2}\left(\mu-\mathrm{N}_{2}\right) \mathrm{Fe}\left({ }^{i} \operatorname{Pr}_{2} \mathrm{Tp}\right)\left(\mathrm{BAr}_{4}{ }_{4}\right)$ & $1.177(5)$ & $1825^{a}$ & 35 \\
\hline$\left(\mathrm{Tp}{ }^{\mathrm{Ph}, \mathrm{Me}} \mathrm{Fe}\right)_{2}\left(\mu-\mathrm{N}_{2}\right)$ & $1.1804(19)$ & $1779^{b}$ & 36 \\
\hline$\left[\mathrm{L}^{t \mathrm{Bu}} \mathrm{Fe}\right]_{2}\left(\mu-\mathrm{N}_{2}\right)$ & $1.182(5)$ & $1778^{b}$ & 37 \\
\hline$\left(\left[{ }^{\mathrm{CY} 5} \mathrm{NpN}^{\mathrm{Me}, \mathrm{Me}}\right] \mathrm{Fe}\right)_{2}\left(\mu-\mathrm{N}_{2}\right)$ & $1.183(6)$ & & 38 \\
\hline$\left(\mathrm{Tp}^{\mathrm{tBu}, \mathrm{Me}} \mathrm{Fe}\right)_{2}\left(\mu-\mathrm{N}_{2}\right)$ & $1.184(7)$ & & 39 \\
\hline$\left[\left({ }^{\mathrm{CY} 5} \mathrm{NpN}^{\mathrm{iPr}, i \mathrm{Pr}{ }^{\prime}}\right) \mathrm{Fe}\right]_{2}\left(\mu-\mathrm{N}_{2}\right)$ & $1.186(3)$ & & 38 \\
\hline$\left[\left(\mathrm{NpNP}^{i \mathrm{Pr}}\right) \mathrm{Fe}\right]_{2}\left(\mu-\mathrm{N}_{2}\right)$ & $1.186(5)$ & $1755^{a}$ & 40 \\
\hline \multirow[t]{2}{*}[(2,6-\mathrm{Me}_{2}\mathrm{Ph})_{2}\text{nacnacCr}(\mu-\mathrm{N}_{2})(\mathrm{THF})]{$_{3}$} & $1.158(4)$ & $2244^{a}$ & 41 \\
\hline & $1.168(4)$ & $2124^{a}$ & \\
\hline$\left[\left({ }^{\mathrm{Ar}} \mathrm{PDI}\right) \mathrm{Cr}(\mathrm{THF})\right]_{2}\left(\mu-\mathrm{N}_{2}\right)$ & $1.241(6)$ & & 42 \\
\hline$\left[\left({ }^{\mathrm{Ar}} \mathrm{PDI}\right) \mathrm{V}\right]_{2}\left(\mu-\mathrm{N}_{2}\right)$ & $1.259(6)$ & & 43 \\
\hline$\left[\left({ }^{\mathrm{Ar}} \mathrm{PDI}\right) \mathrm{V}\right]_{2}\left(\mu-\mathrm{N}_{2}\right)$ & $1.242(5)$ & & 43 \\
\hline$\left[\left(\eta^{5}-\mathrm{C}_{5} \mathrm{Me}_{5}\right) \mathrm{Zr}\right]_{2}\left[\mu-\eta^{2}, \eta^{2}-4,5-\left(\eta^{5}-1-{ }^{i} \mathrm{Pr}-3-\mathrm{Me}-\mathrm{C}_{9} \mathrm{H}_{5}\right)\right]_{2}\left(\mu-\mathrm{N}_{2}\right)$ & $1.197(3)$ & $1563^{a}$ & 44 \\
\hline$\alpha-\mathrm{N}_{2}$ intermediate on $\mathrm{Fe}(111)$ surface & 1.29 & $1490^{a}$ & 45,46 \\
\hline
\end{tabular}

${ }^{a}$ Measured by IR spectroscopy. $\quad{ }^{b}$ Measured by resonance Raman spectroscopy. 
Table S4. Dinitrogen N-N bond lengths and stretching frequencies of PDI-bound iron dinitrogen compounds.

\begin{tabular}{|c|c|c|c|}
\hline complex & N-N $(\AA)$ & $v_{\mathrm{NN}}\left(\mathrm{cm}^{-1}\right)$ & ref \\
\hline$\left({ }^{i \mathrm{Pr}} \mathrm{PDI}\right) \mathrm{Fe}(\mathrm{THT})\left(\mathrm{N}_{2}\right)$ & 1.085 & $2045^{a}$ & 47 \\
\hline ( $\left.{ }^{\mathrm{Ar}} \mathrm{PDI}\right) \mathrm{Fe}\left(\mu-\mathrm{N}_{2}\right) \mathrm{Na}(\mathrm{THF})$ & $1.090(5)$ & & 48 \\
\hline \multirow[t]{2}{*}{$\left({ }^{i \mathrm{Pr}} \mathrm{PDI}\right) \mathrm{Fe}\left(\mathrm{N}_{2}\right)_{2}$} & $1.090(2)$ & $2124^{a}$ & 49 \\
\hline & $1.104(3)$ & $2053^{a}$ & \\
\hline \multirow[t]{2}{*}{$\left(4-\mathrm{Me}_{2} \mathrm{~N}^{-i \mathrm{Pr}} \mathrm{PDI}\right) \mathrm{Fe}\left(\mathrm{N}_{2}\right)_{2}$} & 1.100 & $2034^{a}$ & 50 \\
\hline & 1.133 & & \\
\hline \multirow[t]{2}{*}{$\left(4-{ }^{\mathrm{t}} \mathrm{Bu}-{ }^{i \mathrm{Pr}} \mathrm{PDI}\right) \mathrm{Fe}\left(\mathrm{N}_{2}\right)_{2}$} & 1.112 & & 50 \\
\hline & 1.107 & $2041^{a}$ & \\
\hline \multirow[t]{2}{*}{$\left({ }^{i \mathrm{Pr}} \mathrm{PhPDI}\right) \mathrm{Fe}\left(\mathrm{N}_{2}\right)_{2}$} & $1.106(6)$ & $2138^{a}$ & 51 \\
\hline & $1.107(5)$ & $2086^{a}$ & \\
\hline$\left({ }^{i \mathrm{Pr}} \mathrm{PDI}\right) \mathrm{Fe}\left(\mathrm{PEt}_{3}\right)\left(\mathrm{N}_{2}\right)$ & 1.111 & $2028^{a}$ & 47 \\
\hline 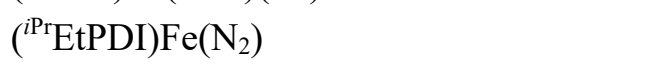 & $1.112(2)$ & $2027^{a}$ & 52 \\
\hline$\left({ }^{i \operatorname{Pr}}{ }_{i} \operatorname{Pr} P D I\right) F e\left(N_{2}\right)$ & $1.117(5)$ & $2026^{a}$ & 52 \\
\hline \multirow[t]{2}{*}[({}^{i\mathrm{Pr},\mathrm{Et}}\mathrm{PDI})\mathrm{Fe}(\mathrm{N}_{2})]{$_{2}\left(\mu-\mathrm{N}_{2}\right)$} & & $2075^{a}$ & 53 \\
\hline & & $2090^{a}$ & \\
\hline$\left[\left({ }^{\mathrm{Me}} \mathrm{BPDI}\right) \mathrm{Fe}\left(\mathrm{N}_{2}\right)\right]_{2}\left(\mu-\mathrm{N}_{2}\right)$ & $1.123(3)$ & & 54 \\
\hline$\left[\left({ }^{\mathrm{Et}} \mathrm{PDI}\right) \mathrm{Fe}\left(\mathrm{N}_{2}\right)\right]_{2}\left(\mu-\mathrm{N}_{2}\right)$ & $1.124(3)$ & & 54 \\
\hline$\left({ }^{i \mathrm{Pr}} \mathrm{PDI}\right) \mathrm{Fe}\left(\mathrm{C}_{6} \mathrm{H}_{5}\right)\left(\mu-\mathrm{N}_{2}\right) \mathrm{Li}\left(\mathrm{OEt}_{2}\right)_{3}$ & & $2069^{a}$ & 55 \\
\hline${ }^{\mathrm{Me}}\left[\mathrm{Fe}_{2} \mathrm{~N}_{2}\right]^{0}$ & $1.135(3)$ & $2003^{a}$ & this work \\
\hline$\left({ }^{i \mathrm{Pr}} \mathrm{BIP}\right) \mathrm{Fe}\left(\mathrm{CH}_{2} \mathrm{CH}_{2} \mathrm{Ph}\right)\left(\mu-\mathrm{N}_{2}\right) \mathrm{MgX}(\mathrm{THF})_{5}$ & $1.137(10)$ & & 56 \\
\hline$\left({ }^{i \mathrm{Pr}} \mathrm{PDI}\right) \mathrm{Fe}\left(\mathrm{CH}_{2} \mathrm{CMe}_{3}\right)\left(\mu-\mathrm{N}_{2}\right) \mathrm{Li}\left(\mathrm{OEt}_{2}\right)_{3}$ & $1.138(3)$ & $1948^{a}$ & 57 \\
\hline$\left({ }^{i \mathrm{Pr}} \mathrm{PDI}\right) \mathrm{Fe}\left(\mathrm{C}_{6} \mathrm{H}_{4}-p-\mathrm{CH}_{3}\right)\left(\mu-\mathrm{N}_{2}\right) \mathrm{Li}\left(\mathrm{OEt}_{2}\right)_{3}$ & $1.138(3)$ & $2068^{a}$ & 55 \\
\hline${ }^{\mathrm{Ph}}\left[\mathrm{Fe}_{2} \mathbf{N}_{2}\right]^{0}$ & $1.139(3)$ & $1959^{a}$ & this work \\
\hline$\left({ }^{\mathrm{Ar}} \mathrm{PDI}\right) \mathrm{Fe}\left(\mu-\mathrm{N}_{2}\right) \mathrm{Na}\left(\mathrm{OEt}_{2}\right)_{3}$ & $1.154(6)$ & & 48 \\
\hline
\end{tabular}

${ }^{a}$ Measured by IR spectroscopy.

Table S5. Dinitrogen N-N bond lengths and stretching frequencies of alkali metal-containing di-iron dinitrogen compounds.

\begin{tabular}{l|c|c|c|}
\multicolumn{1}{c|}{ complex } & $\mathrm{N}-\mathrm{N}(\AA)$ & $v_{\mathrm{NN}}\left(\mathrm{cm}^{-1}\right)$ & ref \\
{$\left[\mathrm{Na}(\mathrm{THF})_{6}\right]\left\{\left[\left(\mathrm{PhBP}^{i \mathrm{Pr}_{3}}\right) \mathrm{Fe}\right]_{2}\left(\mu-\mathrm{N}_{2}\right)\right\}$} & $1.171(4)$ & & 58 \\
{$\left[\mathrm{~K}(18 \text {-crown-6)(THF })_{2}\right]\left[\left(\mathrm{L}^{\mathrm{Me}} \mathrm{Fe}\right)_{2}\left(\mu-\mathrm{N}_{2}\right)\right]$} & $1.186(6)$ & $1749^{a}$ & 59 \\
{$\left[\mathrm{~K}(18 \text {-crown-6)(12-crown-4) }]_{2}\left[\left(\mathrm{~L}^{\mathrm{Me}} \mathrm{Fe}\right)_{2}\left(\mu-\mathrm{N}_{2}\right)\right]\right.$} & $1.190(8)$ & $1738^{a}$ & 60 \\
$\mathrm{Rb}_{2}\left[\mathrm{LFe}\left(\mu-\mathrm{N}_{2}\right)\right]_{3}$ & $1.191(14)$ & & 61 \\
$\mathrm{Cs}_{2}\left[\mathrm{LFe}\left(\mu-\mathrm{N}_{2}\right)\right]_{3}$ & 1.199 & & 61 \\
$\mathrm{~K}_{2}\left[\left(\mathrm{~L}^{t \mathrm{Bu}} \mathrm{Fe}\right)_{2}\left(\mu-\mathrm{N}_{2}\right)\right]$ & $1.233(6)$ & $1589^{a}$ & 62 \\
$\mathrm{Cs}_{2}\left[(\mathrm{LFe})_{3}(\mu-\mathrm{Cl})\left(\mu-\mathrm{N}_{2}\right)_{2}\right]$ & $1.234(11)$ & & 61 \\
$\mathrm{Na}_{2}\left[\left(\mathrm{~L}{ }^{\mathrm{Me}} \mathrm{Fe}\right)_{2}\left(\mu-\mathrm{N}_{2}\right)\right]$ & $1.253(6)$ & $1612^{a}$ & 63 \\
$\mathrm{Rb}_{2}\left[\left(\mathrm{~L}{ }^{\mathrm{Me}} \mathrm{Fe}\right)_{2}\left(\mu-\mathrm{N}_{2}\right)\right]$ & $1.257(8)$ & $1621^{a}$ & 63 \\
$\mathrm{Cs}_{2}\left[\left(\mathrm{~L}^{\mathrm{Me}} \mathrm{Fe}\right)_{2}\left(\mu-\mathrm{N}_{2}\right)\right]$ & $1.33(2)$ & $1613^{a}$ & 63
\end{tabular}

${ }^{a}$ Measured by IR spectroscopy. 


\section{Spectroscopic Data}

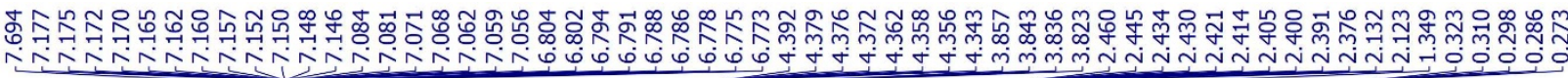

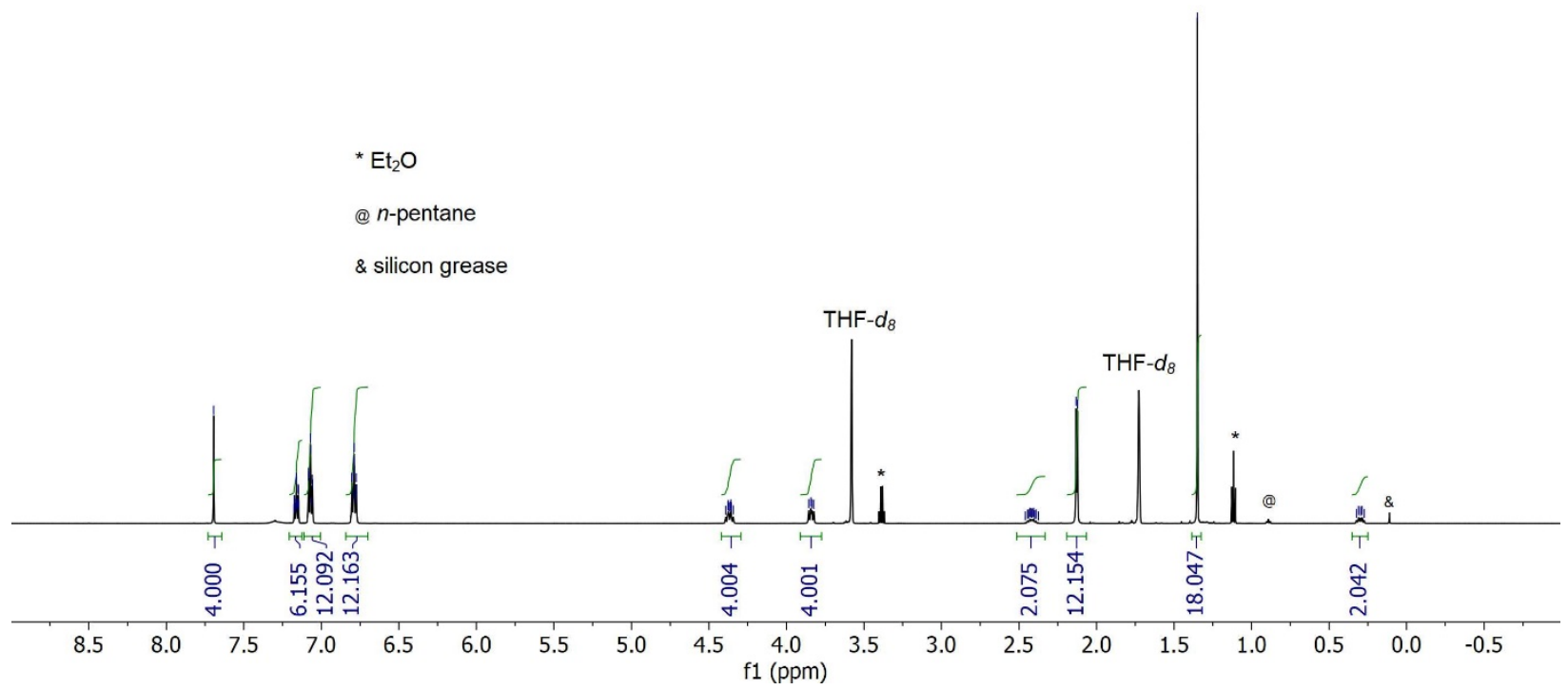

Figure S3. ${ }^{1} \mathrm{H}$ NMR spectrum of ${ }^{\mathbf{P h}}\left[\mathbf{F e}_{2} \mathbf{N}_{2}\right]^{\mathbf{0}}$ in THF- $d_{8}(298 \mathrm{~K})$.

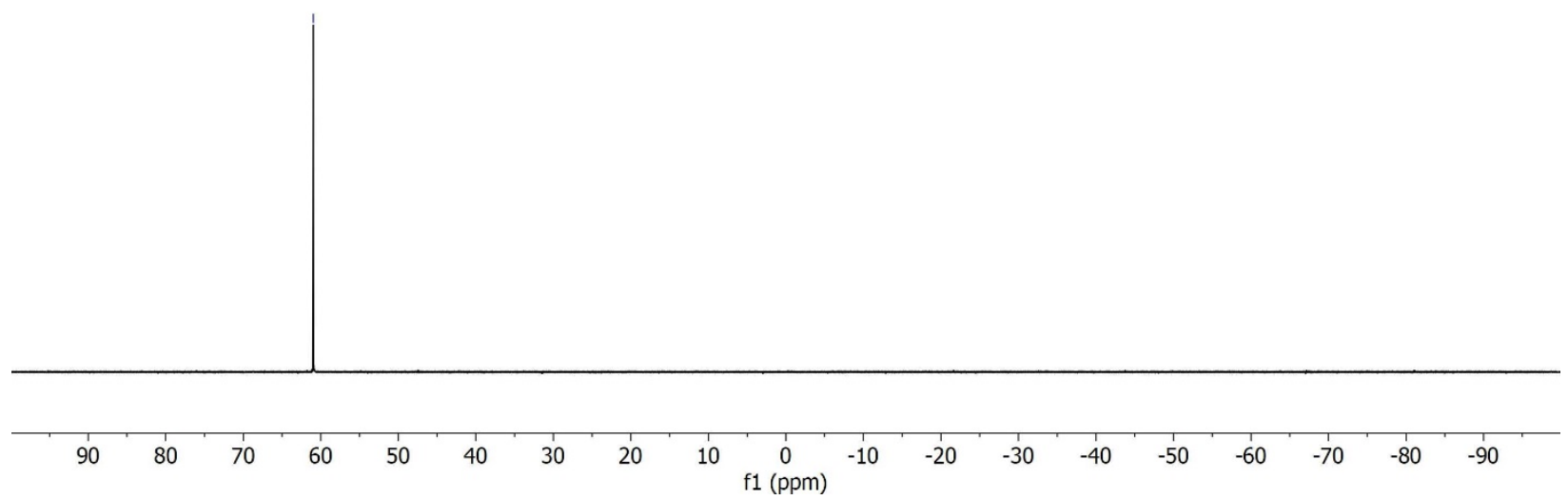

Figure S4. ${ }^{31} \mathrm{P}\left\{{ }^{1} \mathrm{H}\right\}$ NMR spectrum of ${ }^{\mathbf{P h}}\left[\mathbf{F e}_{2} \mathbf{N}_{2}\right]^{\mathbf{0}}$ in THF- $d_{8}(298 \mathrm{~K})$. 


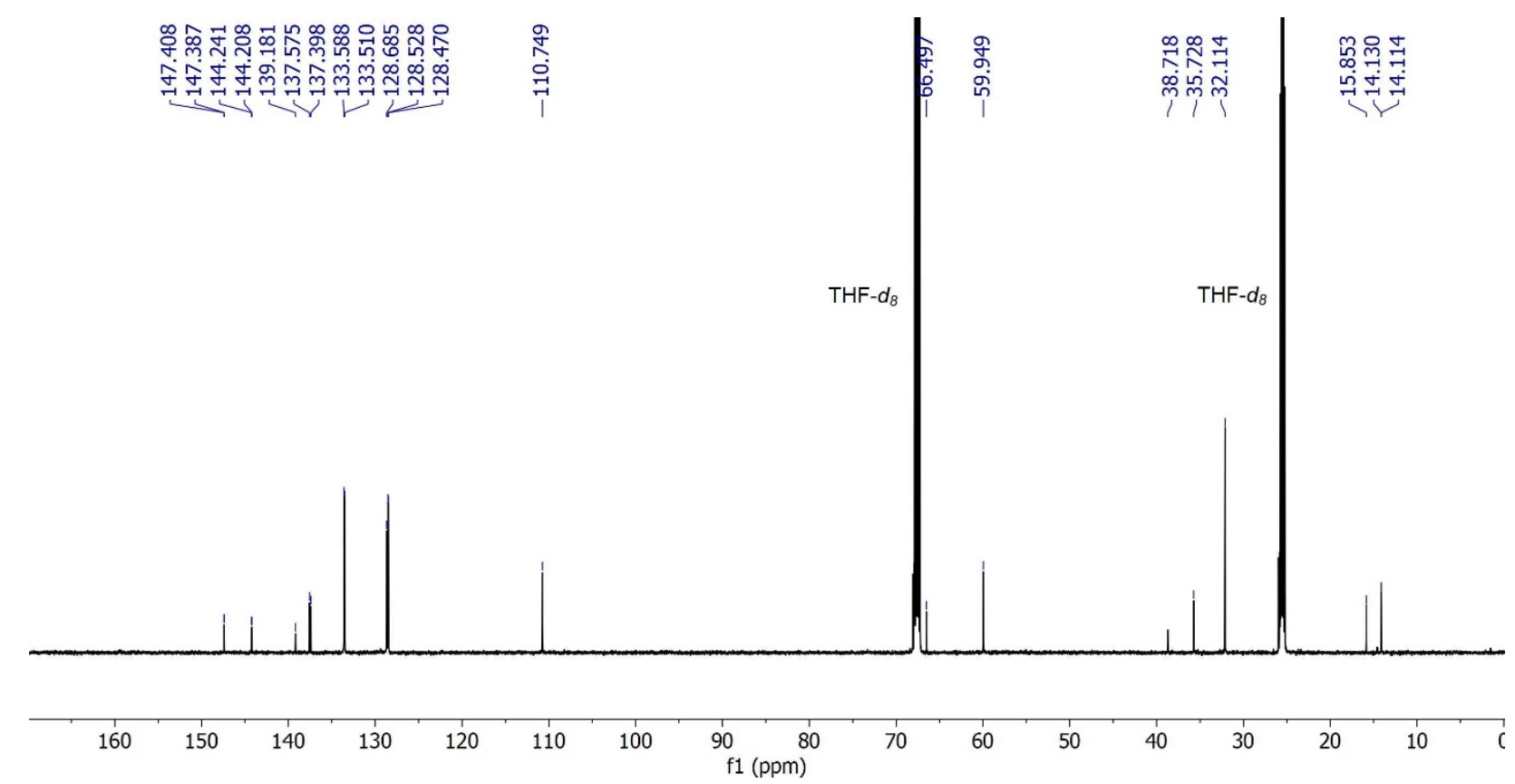

Figure S5. ${ }^{13} \mathrm{C}\left\{{ }^{1} \mathrm{H}\right\}$ NMR spectrum of ${ }^{\mathbf{P h}}\left[\mathbf{F e}_{2} \mathbf{N}_{2}\right]^{\mathbf{0}}$ in THF- $d_{8}(298 \mathrm{~K})$.

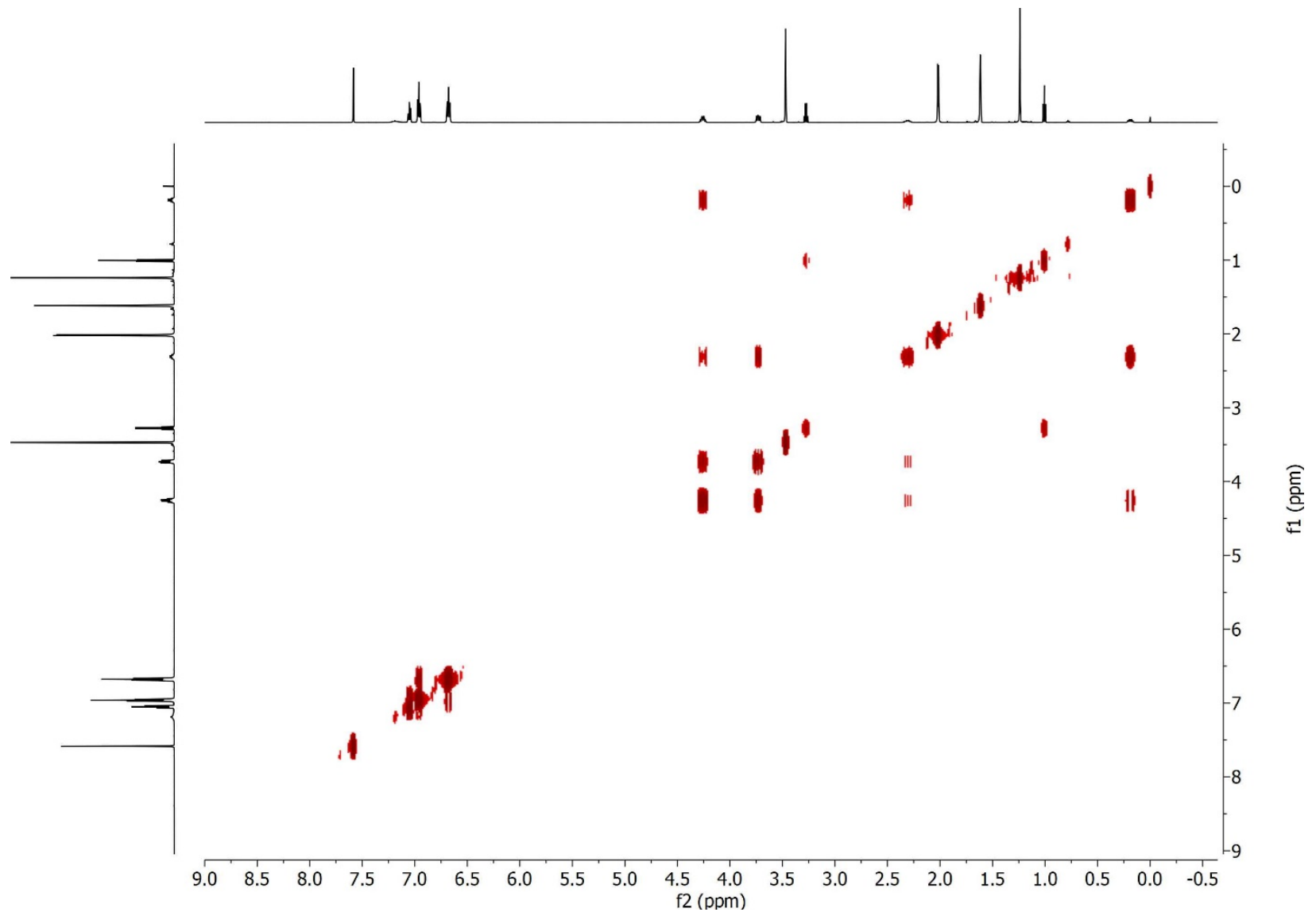

Figure S6. ${ }^{1} \mathrm{H}-{ }^{1} \mathrm{H}$ COSY NMR spectrum of ${ }^{\mathbf{P h}}\left[\mathbf{F e}_{2} \mathbf{N}_{2}\right]^{\mathbf{0}}$ in THF- $d_{8}(298 \mathrm{~K})$. 


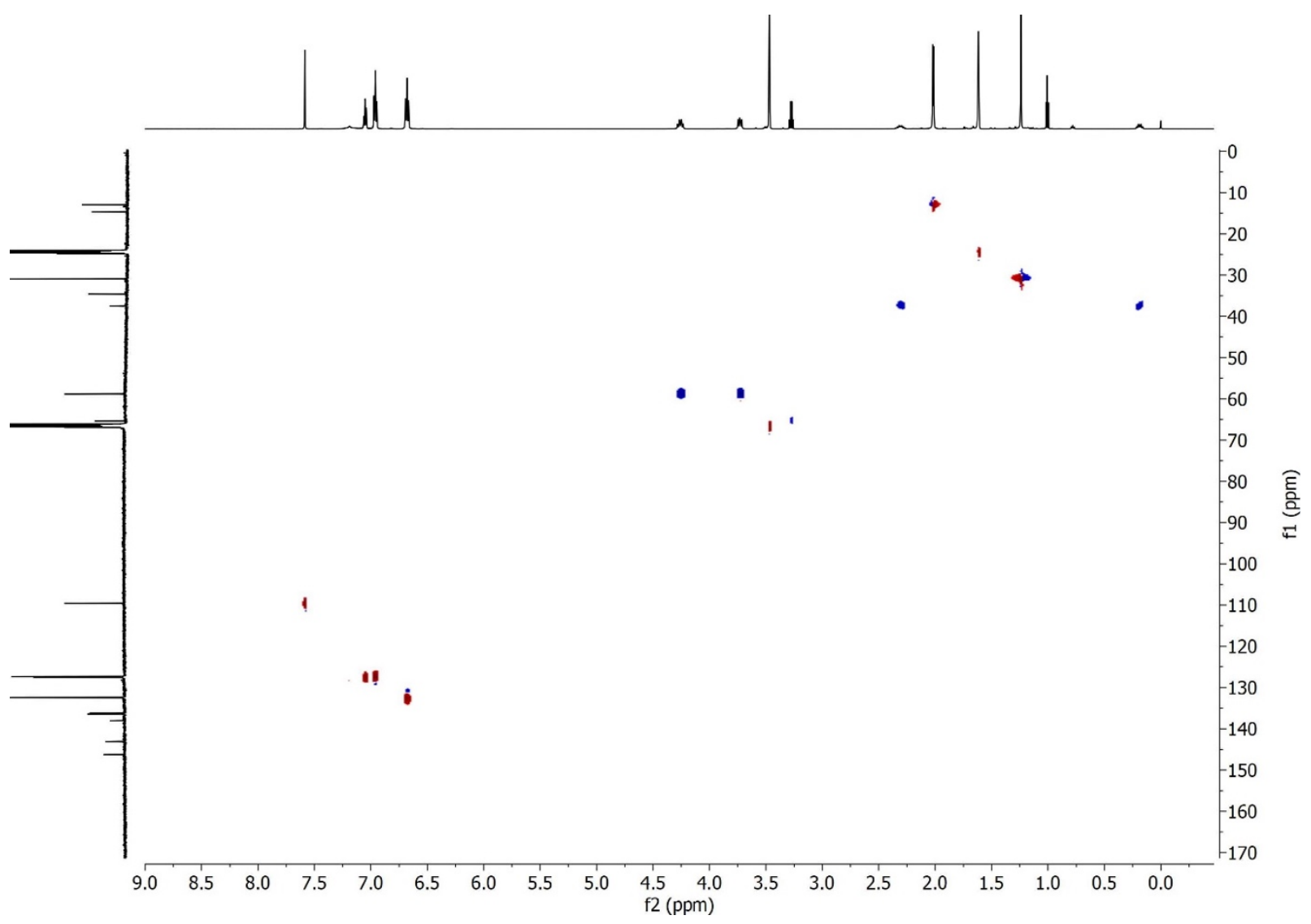

Figure S7. ${ }^{1} \mathrm{H}-{ }^{13} \mathrm{C}$ HSQC NMR spectrum of ${ }^{\mathbf{P h}}\left[\mathbf{F e}_{2} \mathbf{N}_{2}\right]^{\mathbf{0}}$ in THF- $d_{8}(298 \mathrm{~K})$.

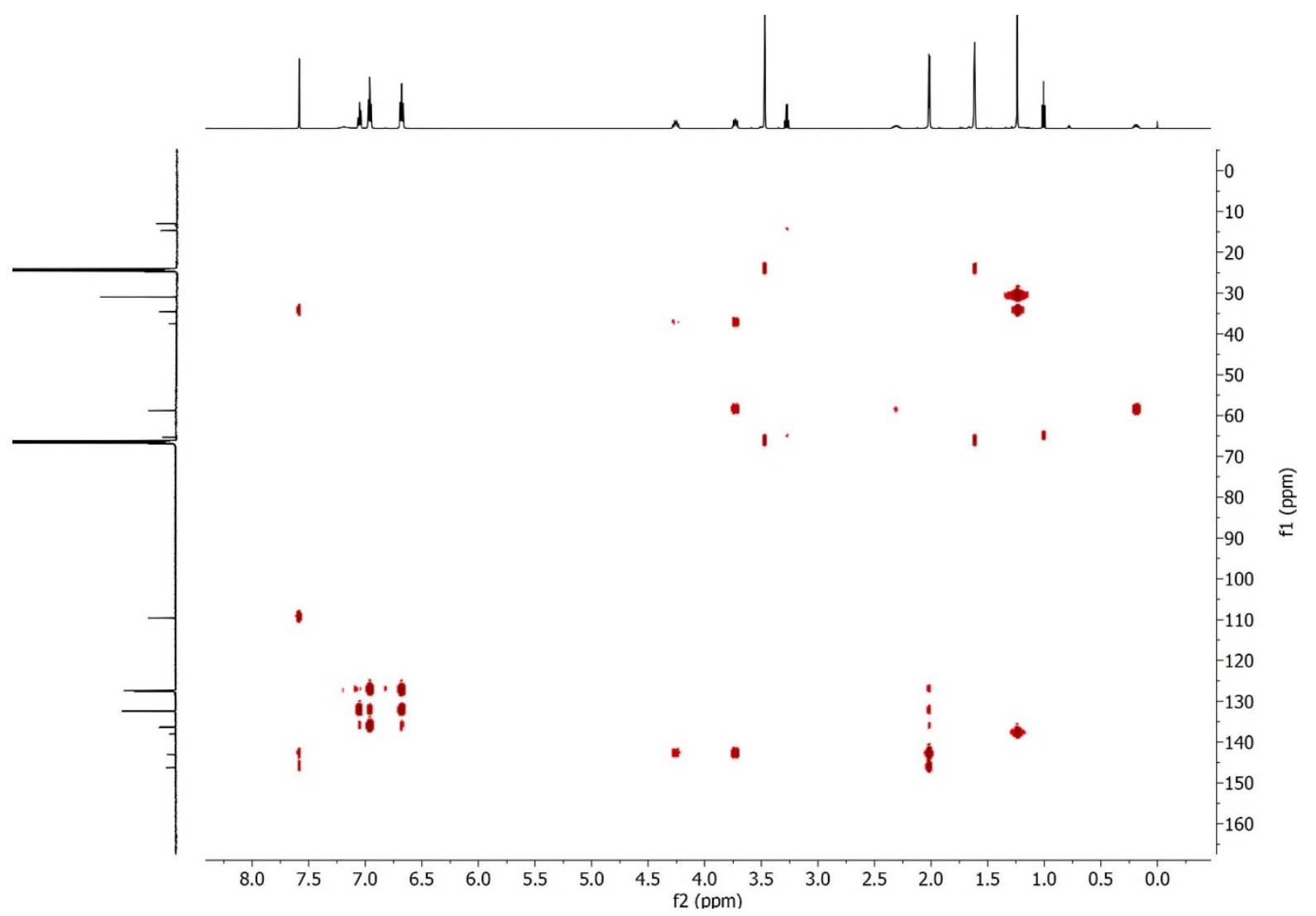

Figure S8. ${ }^{1} \mathrm{H}-{ }^{13} \mathrm{C}$ HMBC NMR spectrum of ${ }^{\mathbf{P h}}\left[\mathbf{F e}_{2} \mathbf{N}_{2}\right]^{\mathbf{0}}$ in THF- $d_{8}(298 \mathrm{~K})$. 


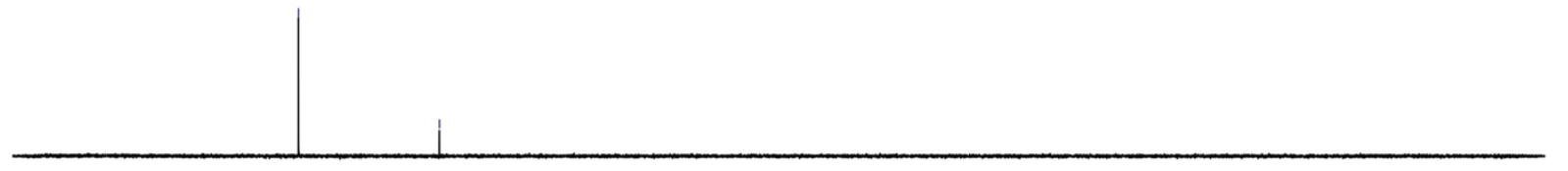

250

200

150

100

$50 \quad 0$

Figure S9. ${ }^{15} \mathrm{~N}$ NMR spectrum of ${ }^{\mathbf{P h}}\left[\mathbf{F e}_{2}{ }^{15} \mathbf{N}_{2}\right]^{\mathbf{0}}$ in THF- $d_{8}(298 \mathrm{~K})$. The feature at 309.7 ppm was tentatively assigned as free ${ }^{15} \mathrm{~N}_{2} \cdot{ }^{15}$
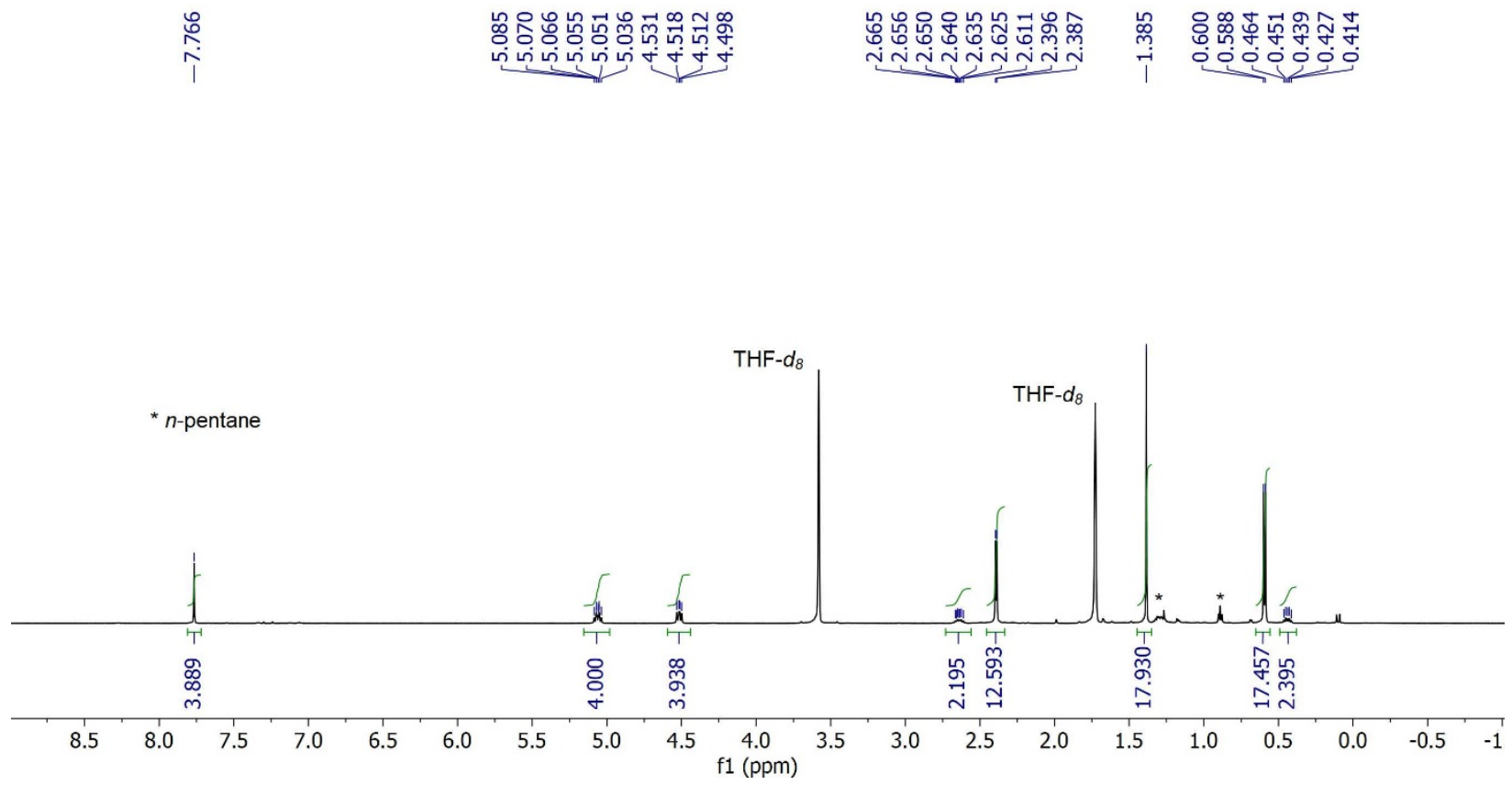

Figure S10. ${ }^{1} \mathrm{H}$ NMR spectrum of ${ }^{\mathbf{M e}}\left[\mathbf{F e}_{2} \mathbf{N}_{2}\right]^{\mathbf{0}}$ in THF- $d_{8}(298 \mathrm{~K})$. 


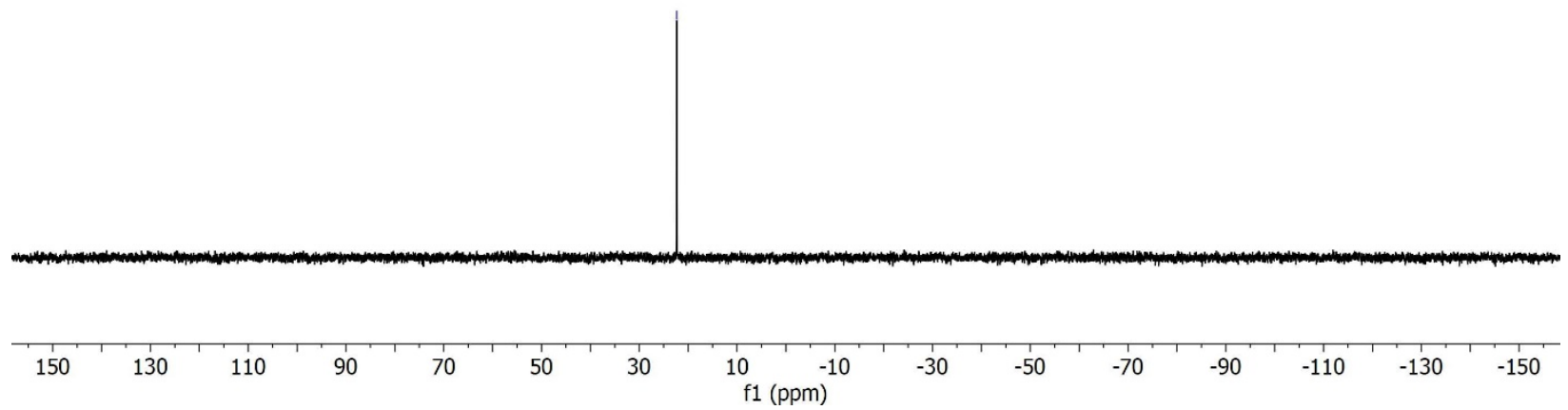

Figure S11. ${ }^{31} \mathrm{P}\left\{{ }^{1} \mathrm{H}\right\}$ NMR spectrum of ${ }^{\mathbf{M e}}\left[\mathbf{F e}_{2} \mathbf{N}_{2}\right]^{\mathbf{0}}$ in THF- $d_{8}(298 \mathrm{~K})$.

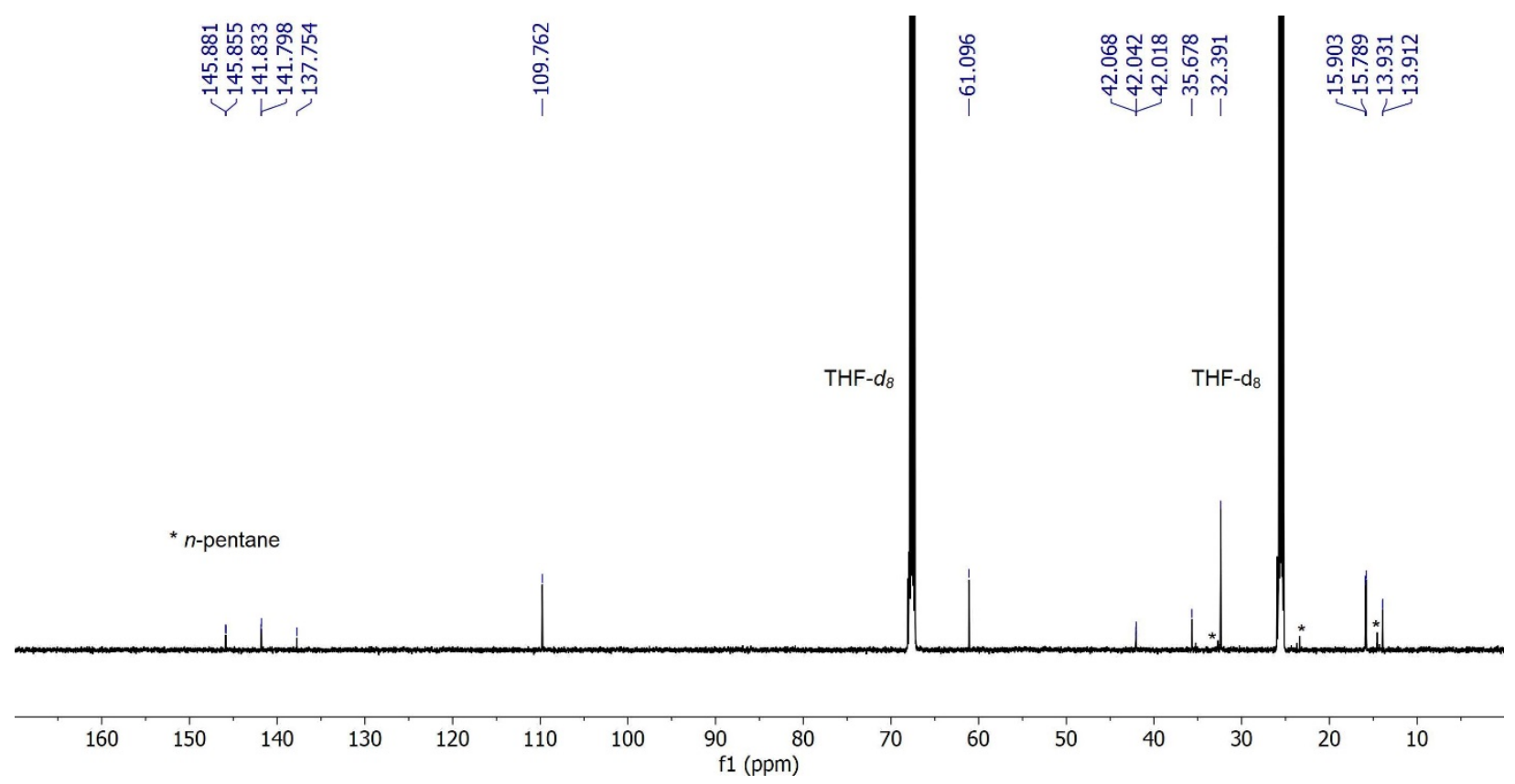

Figure S12. ${ }^{13} \mathrm{C}\left\{{ }^{1} \mathrm{H}\right\}$ NMR spectrum of ${ }^{\mathbf{M e}}\left[\mathbf{F} \mathbf{e}_{2} \mathbf{N}_{2}\right]^{\mathbf{0}}$ in THF- $d_{8}(298 \mathrm{~K})$. 


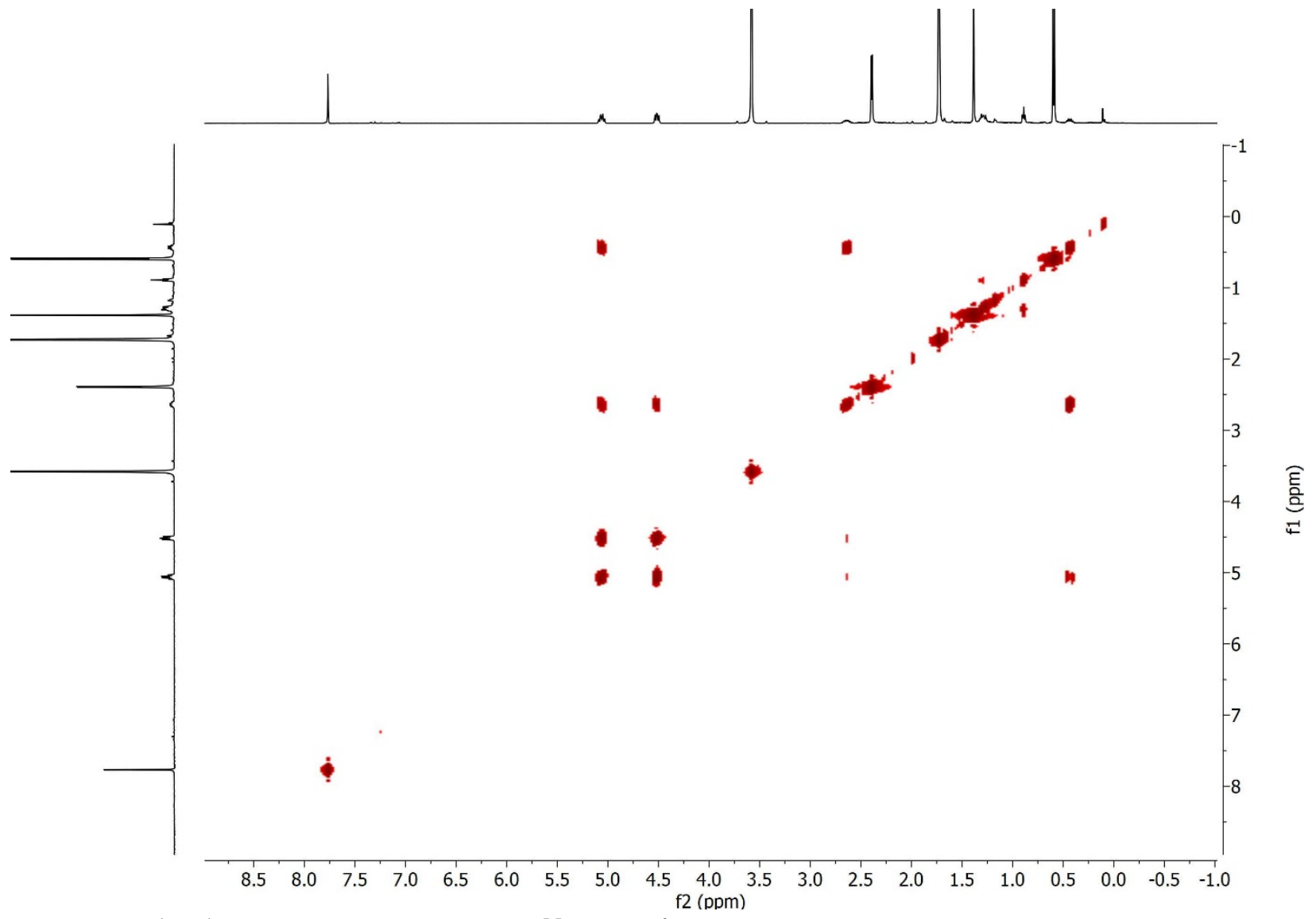

Figure S13. ${ }^{1} \mathrm{H}-{ }^{1} \mathrm{H}$ COSY NMR spectrum of ${ }^{\mathrm{Me}}\left[\mathbf{F e}_{2} \mathbf{N}_{2}\right]^{\mathbf{0}}$ in THF- $d_{8}(298 \mathrm{~K})$.

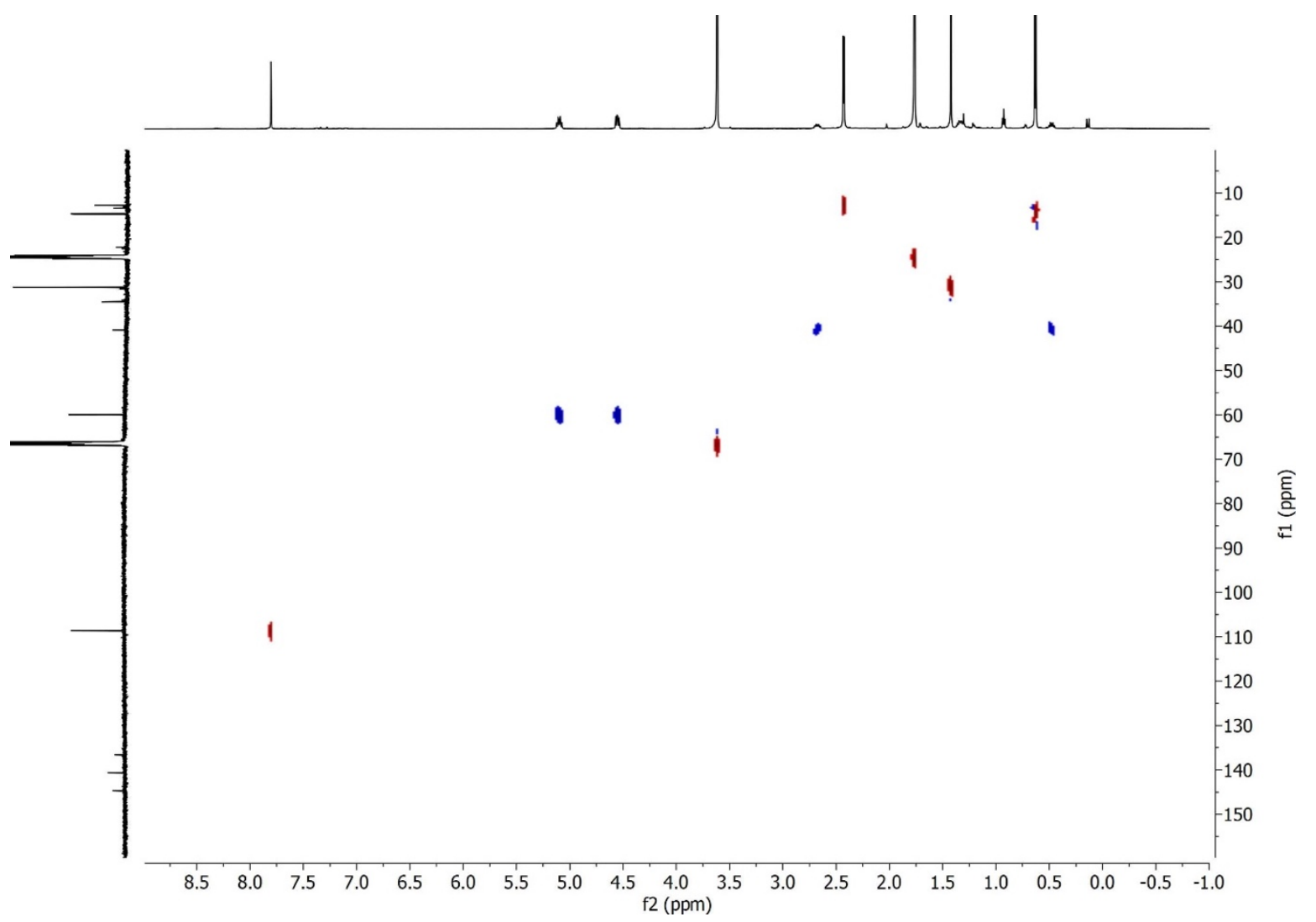

Figure S14. ${ }^{1} \mathrm{H}-{ }^{13} \mathrm{C}$ HSQC NMR spectrum of ${ }^{\mathrm{Me}}\left[\mathbf{F e}_{2} \mathbf{N}_{2}\right]^{\mathbf{0}}$ in THF- $d_{8}(298 \mathrm{~K})$. 


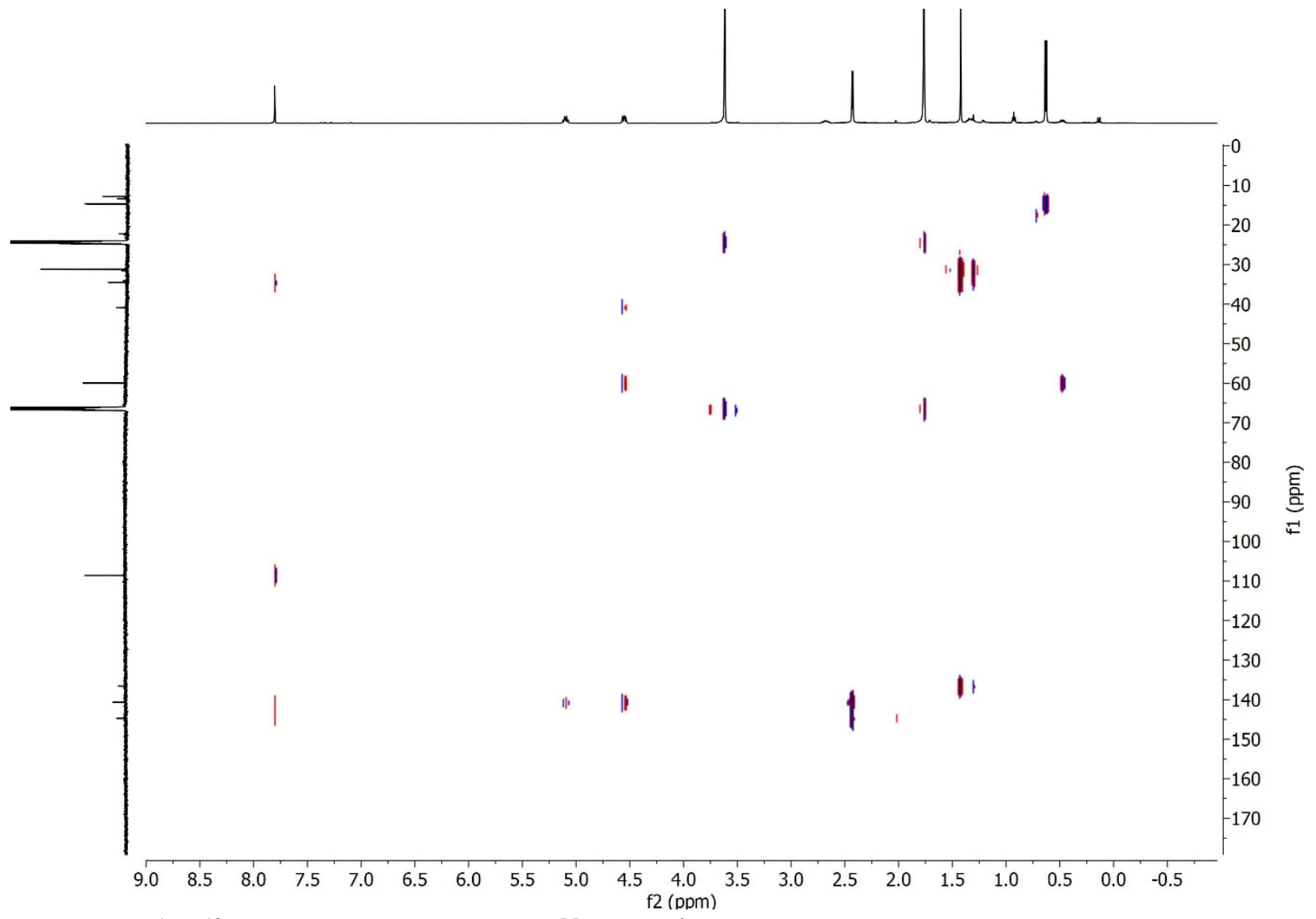

Figure S15. ${ }^{1} \mathrm{H}-{ }^{13} \mathrm{C}$ HMBC NMR spectrum of ${ }^{\mathbf{M e}}\left[\mathbf{F e}_{2} \mathbf{N}_{2}\right]^{\mathbf{0}}$ in THF- $d_{8}(298 \mathrm{~K})$.

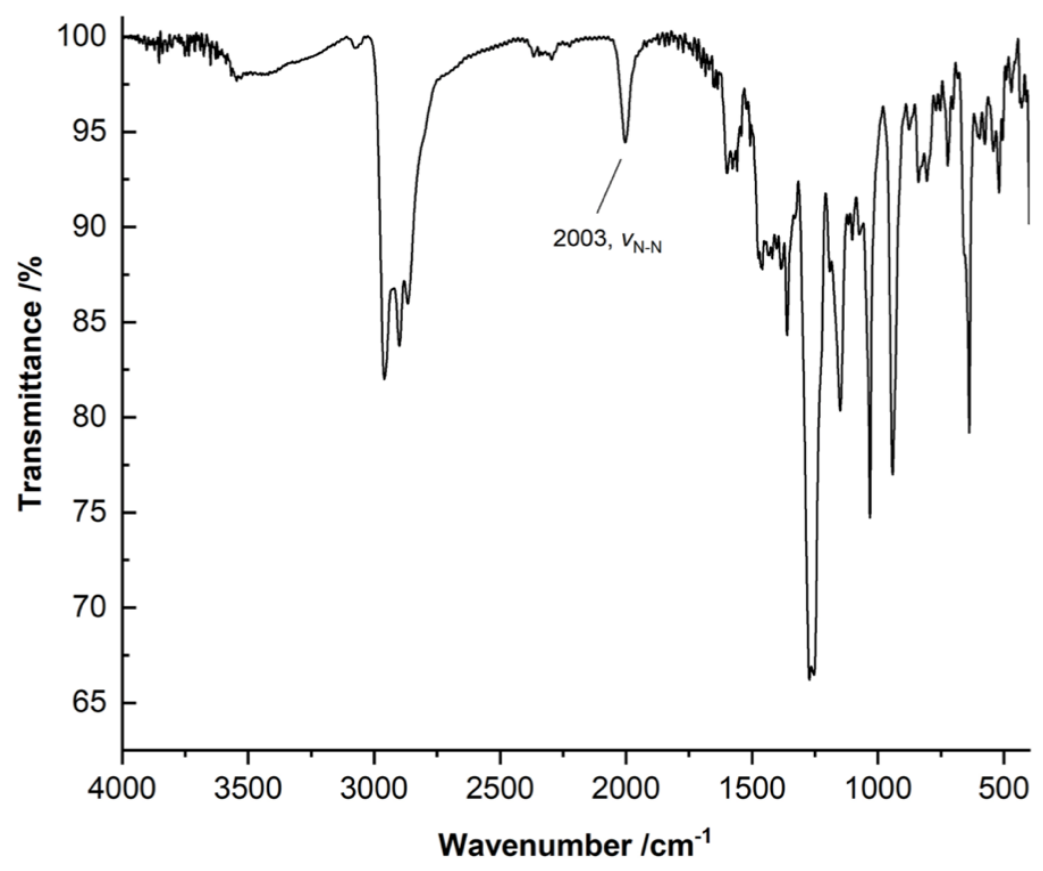

Figure S16. FT-IR spectra of ${ }^{\mathrm{Me}}\left[\mathbf{F e}_{2} \mathbf{N}_{2}\right]^{0}$ (KBr pellet). 


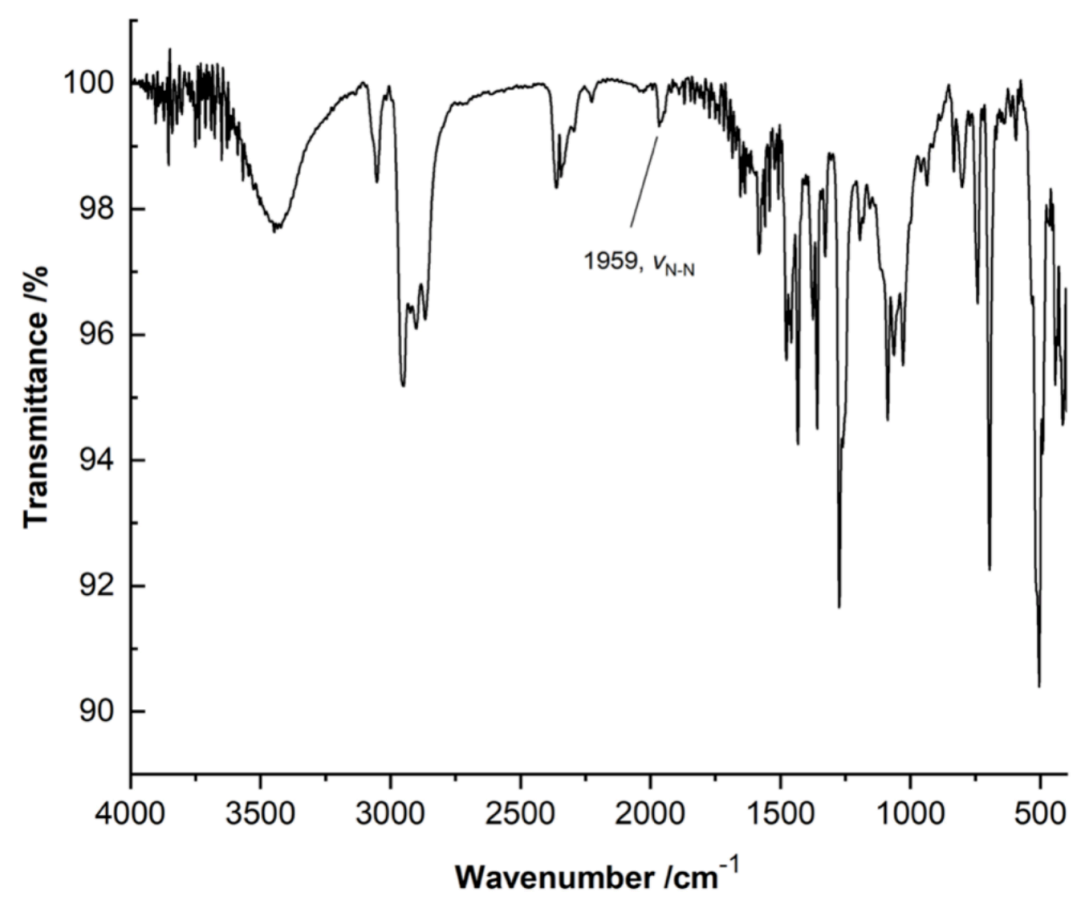

Figure S17. FT-IR spectra of ${ }^{\mathrm{Ph}}\left[\mathrm{Fe}_{2} \mathbf{N}_{2}\right]^{0}$ (KBr pellet).

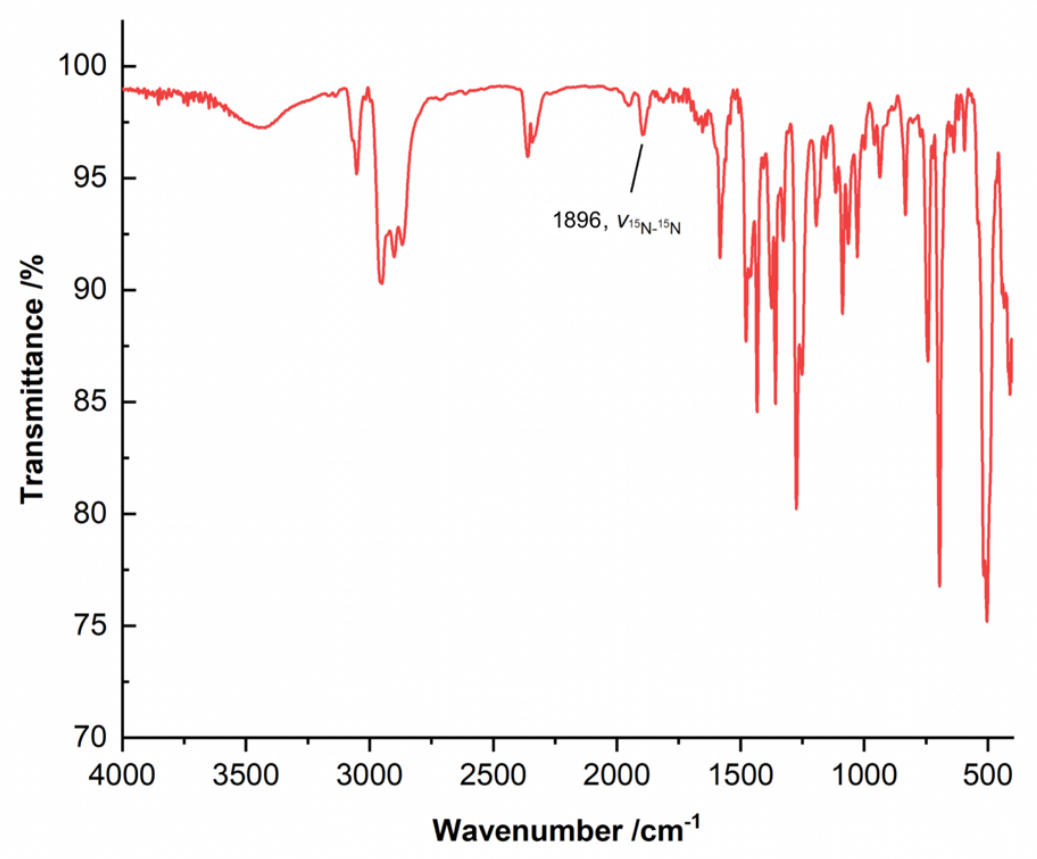

Figure S18. FT-IR spectra of ${ }^{\mathrm{Ph}}\left[\mathbf{F e}_{2}{ }^{15} \mathbf{N}_{2}\right]^{0}$ (KBr pellet). 


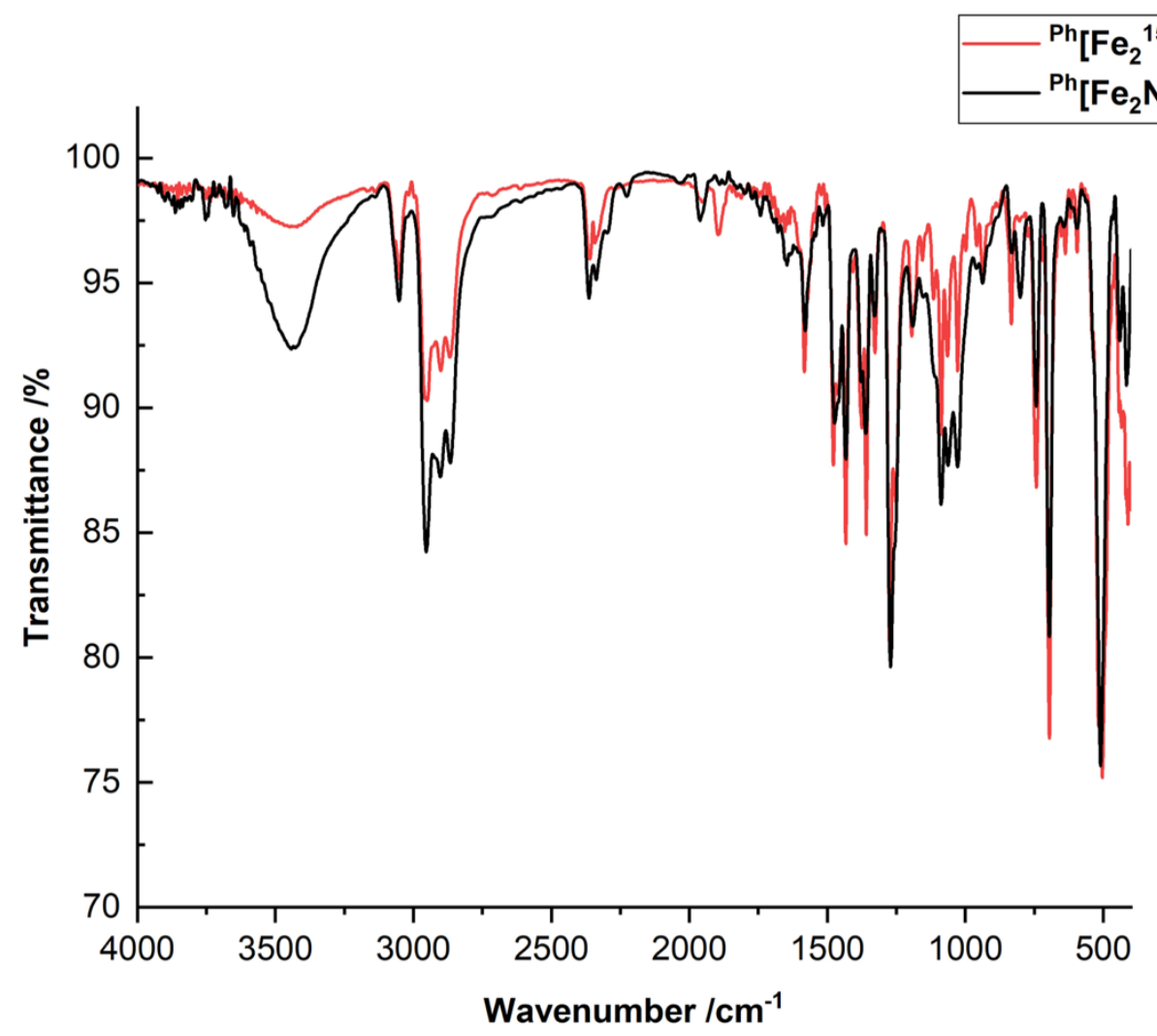

Figure S19. Overlay of the FT-IR spectra of ${ }^{\mathrm{Ph}}\left[\mathrm{Fe}_{2} \mathbf{N}_{2}\right]^{0}$ (black line) and ${ }^{\mathrm{Ph}}\left[\mathrm{Fe}_{2}{ }^{15} \mathbf{N}_{2}\right]^{0}$ (red line).

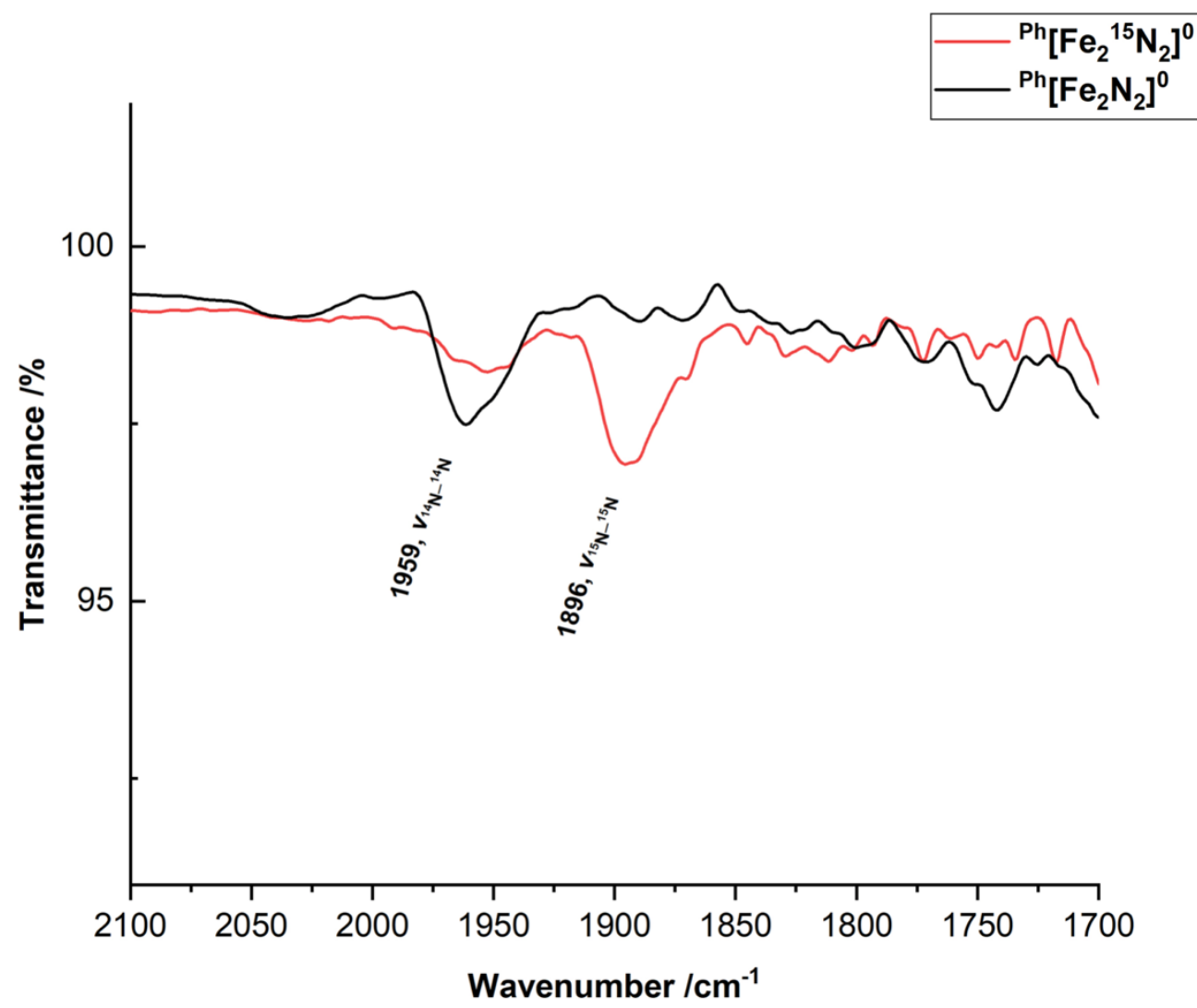

Figure S20. Selected region of the FT-IR spectra of ${ }^{\mathrm{Ph}}\left[\mathbf{F e}_{2} \mathbf{N}_{2}\right]^{0}$ (black line) and ${ }^{\mathrm{Ph}}\left[\mathrm{Fe}_{2}{ }^{15} \mathbf{N}_{2}\right]^{0}$ (red line). 


\section{Electrochemistry Data}
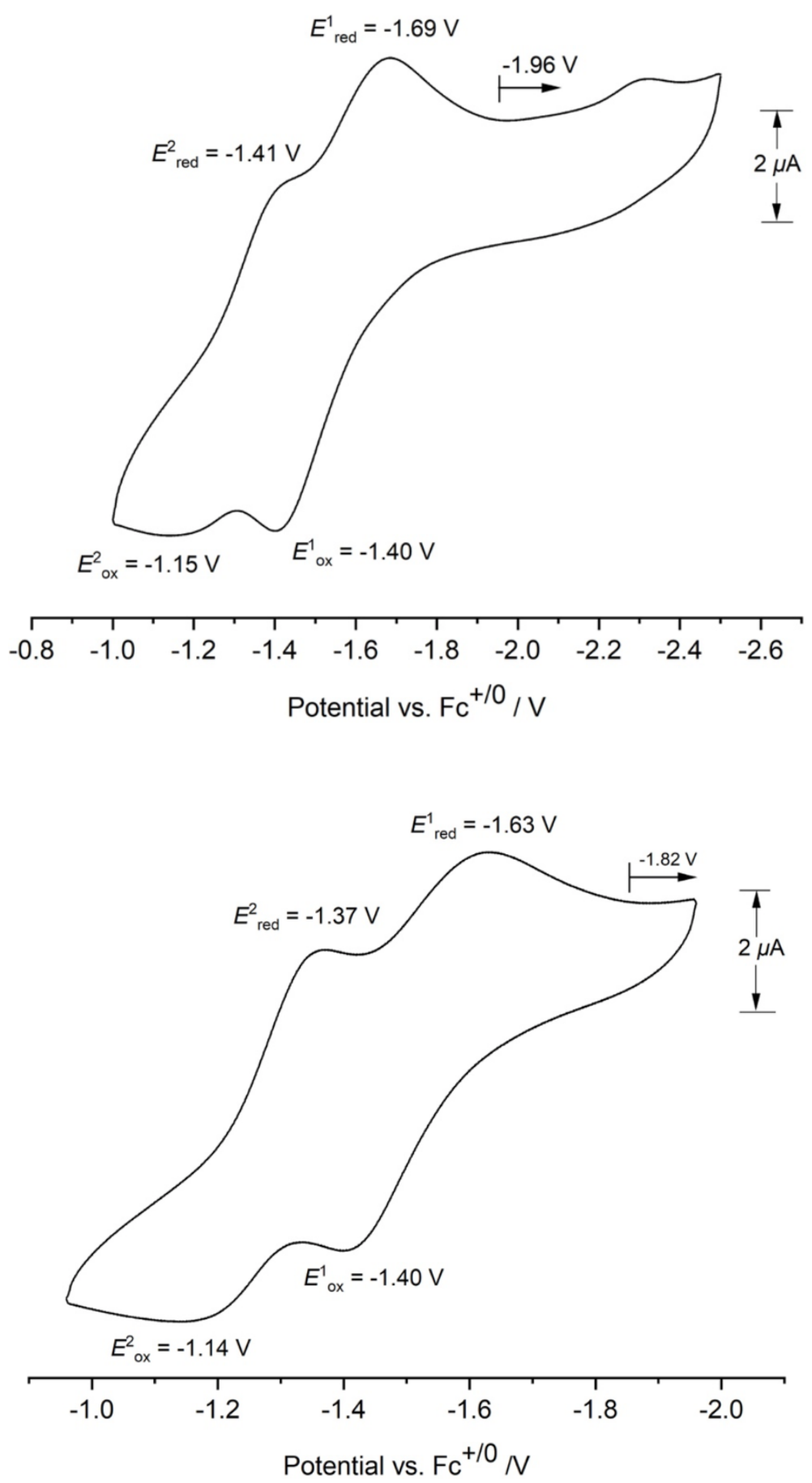

Figure S21. Cyclic voltammograms of ${ }^{\mathrm{Ph}}\left[\mathrm{Fe}_{2} \mathbf{N}_{2}\right]^{0}(1 \mathrm{mM}$ in THF), using a glassy carbon (3 mm outer diameter) working electrode, a platinum wire counter electrode, a $\mathrm{AgPF}_{6}(100 \mathrm{mM}$ in THF $) / \mathrm{Ag}$ reference electrode and $100 \mathrm{mM}\left[{ }^{n} \mathrm{Bu}_{4} \mathrm{~N}\right]\left[\mathrm{PF}_{6}\right]$ as the supporting electrolyte. Scan rate $100 \mathrm{mV} / \mathrm{s}$ in THF versus $\mathrm{Cp}_{2} \mathrm{Fe}^{+/ 0}$. The plots show one trace from multi-cycle sweeps. 


\section{Computational Results}

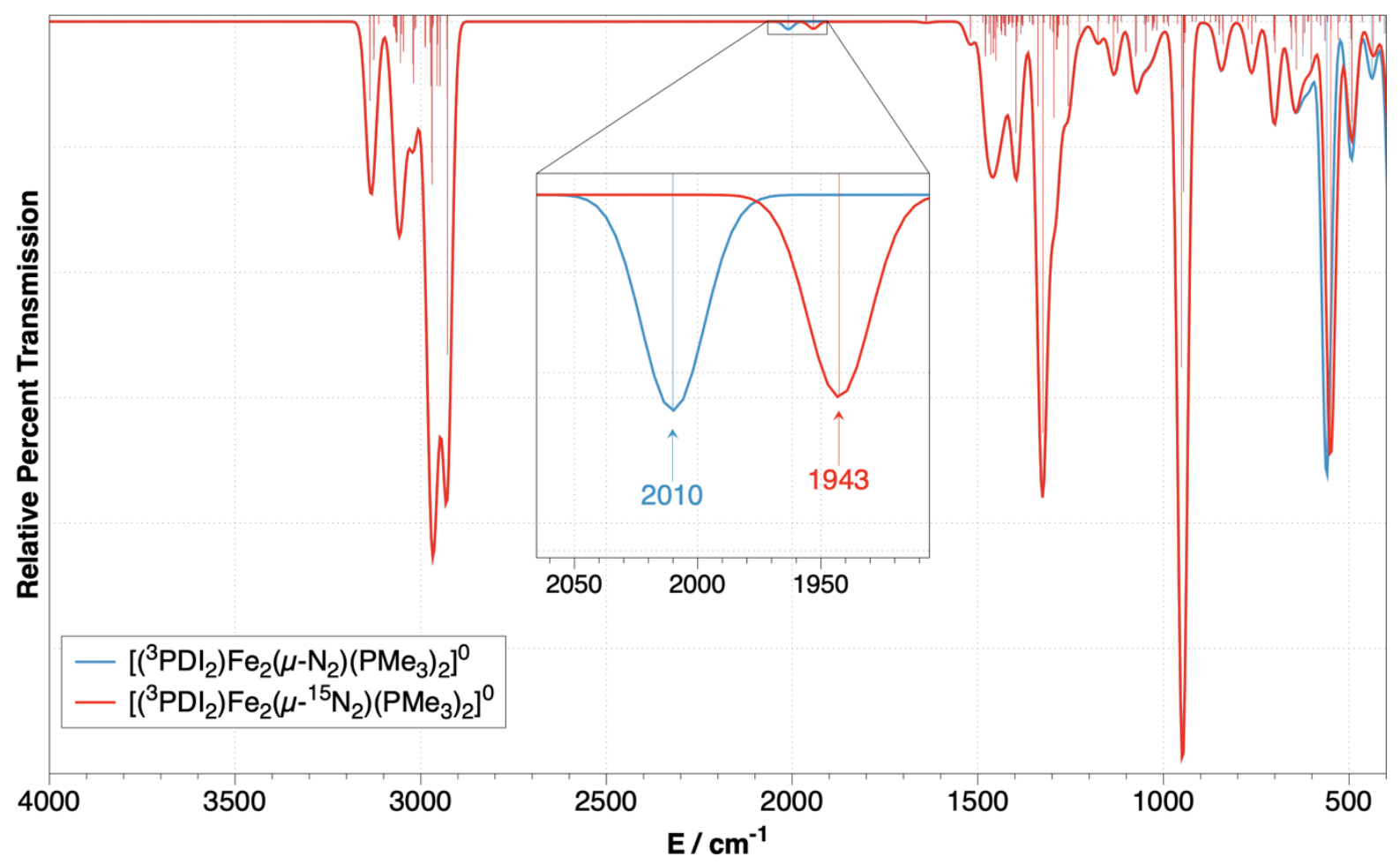

Figure S22. Calculated IR spectra of the truncated versions of ${ }^{\mathrm{Me}}\left[\mathrm{Fe}_{2} \mathbf{N}_{2}\right]^{0}$ (red) and ${ }^{\mathrm{Me}}\left[\mathrm{Fe}_{2}{ }^{15} \mathbf{N}_{2}\right]^{\mathbf{0}}$ (blue). 


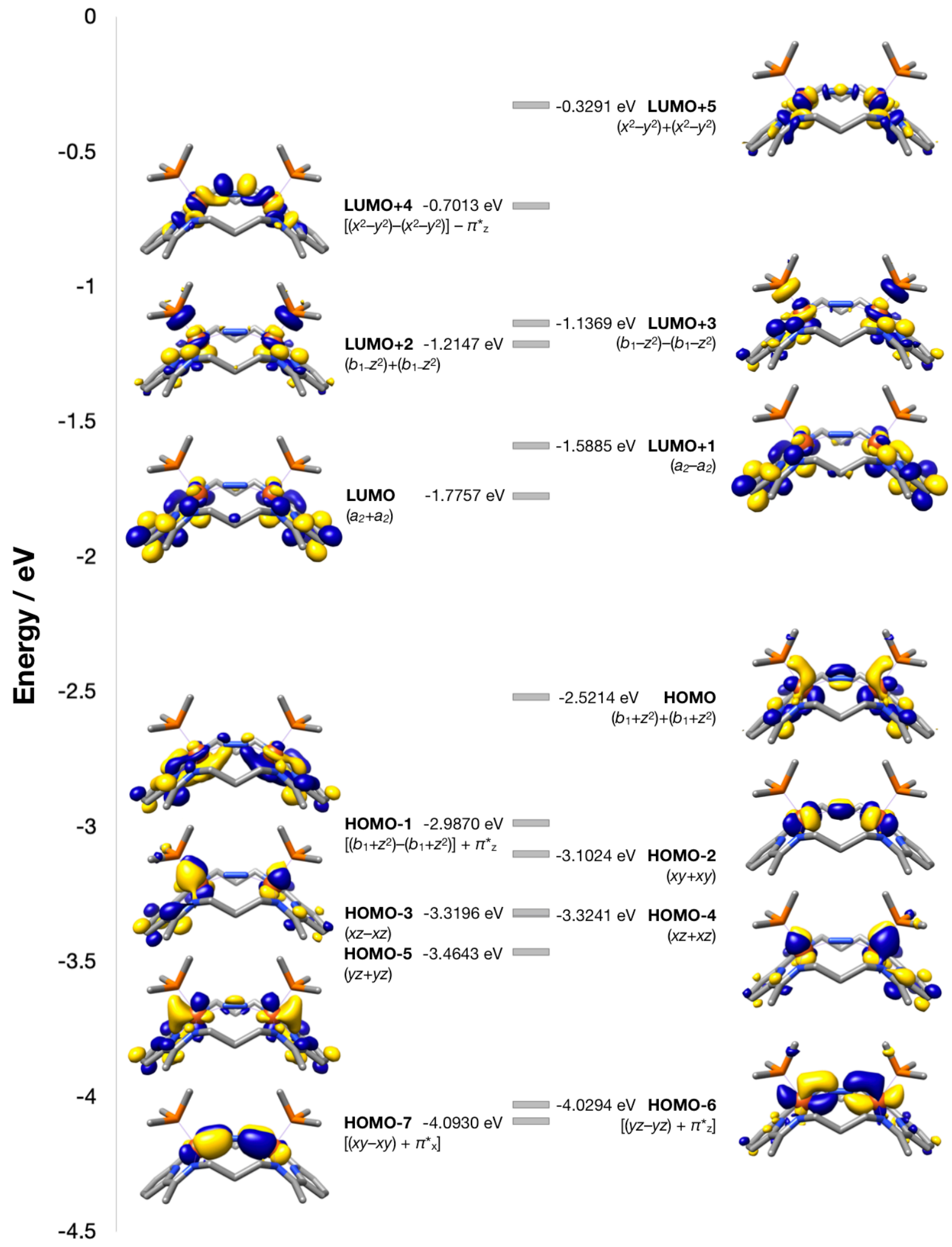

Figure S23. Quantitative molecular orbital diagram for the truncated version of ${ }^{\mathrm{Me}}\left[\mathrm{Fe}_{2} \mathbf{N}_{2}\right]^{0}$. 
DFT-optimized Atomic Coordinates

\begin{tabular}{|c|c|c|c|}
\hline \multicolumn{4}{|c|}{$\left[\left({ }^{3} \mathrm{PDI}_{2}\right) \mathrm{Fe}_{2}\left(\mu-\mathrm{N}_{2}\right)\left(\mathrm{PMe}_{3}\right)_{2}\right]^{0}\left({ }^{\mathrm{Me}}\left[\mathbf{F e}_{2} \mathbf{N}_{2}\right]^{0}\right)$} \\
\hline $\mathrm{Fe}$ & 0.00514284296710 & 0.02626930822387 & 0.14266483149390 \\
\hline $\mathrm{Fe}$ & -0.06816894117977 & 4.56167826172399 & 0.16344805053431 \\
\hline $\mathrm{P}$ & -0.37018056654390 & -1.16330925522904 & 1.88112467407858 \\
\hline $\mathrm{P}$ & -0.47469736751201 & 5.72651294470157 & 1.91173453749653 \\
\hline $\mathrm{N}$ & -0.15841655019757 & 1.71516593522101 & 0.74967440405158 \\
\hline $\mathrm{N}$ & -0.17741815081335 & 2.86292081967652 & 0.75599841771712 \\
\hline $\mathrm{N}$ & 0.31598104977411 & -1.29278642517028 & -1.12772225131666 \\
\hline $\mathrm{N}$ & -1.76206253859371 & -0.05979170020905 & -0.70628376569397 \\
\hline $\mathrm{N}$ & 1.96815135809168 & -0.00165393822731 & 0.14483464317672 \\
\hline $\mathrm{N}$ & 0.19724741174357 & 5.89944884805212 & -1.09739233402911 \\
\hline $\mathrm{N}$ & -1.83993880282515 & 4.59714534105396 & -0.68211816186483 \\
\hline $\mathrm{N}$ & 1.89210219001449 & 4.65111376962551 & 0.16089771780160 \\
\hline $\mathrm{C}$ & -0.76647833438930 & -1.82134262333615 & -1.83454063357159 \\
\hline $\mathrm{C}$ & 1.61251231457268 & -1.78614439245016 & -1.28882637459707 \\
\hline $\mathrm{C}$ & -2.80366830220681 & 0.941797 & -0.441363 \\
\hline $\mathrm{H}$ & -2.76622452159615 & 1.15603114822899 & 0.64315825811725 \\
\hline $\mathrm{H}$ & -3.81842369762358 & 0.55447493481696 & -0.65974735573477 \\
\hline $\mathrm{C}$ & 1.02158882195223 & -2.26383131998319 & 2.37236664902976 \\
\hline $\mathrm{H}$ & 1.36171012384152 & -2.82374531072882 & 1.48042775749777 \\
\hline $\mathrm{H}$ & 0.72928159236800 & -2.97535466733415 & 3.17081970936185 \\
\hline $\mathrm{H}$ & 1.86374713481268 & -1.63819628108589 & 2.72253814060455 \\
\hline $\mathrm{C}$ & 3.92967241316430 & 5.94026981895486 & -0.56746402989205 \\
\hline $\mathrm{H}$ & 4.34342424740809 & 6.14841071485082 & 0.44323970329991 \\
\hline $\mathrm{H}$ & 4.15472156558272 & 6.81677149427390 & -1.20124826727513 \\
\hline $\mathrm{H}$ & 4.50160904909710 & 5.08202105034627 & -0.98093544881583 \\
\hline $\mathrm{C}$ & -3.27574132716554 & -1.32190183303680 & -2.27417090719902 \\
\hline $\mathrm{H}$ & -4.08379860584768 & -1.57324394619186 & -1.55290911025568 \\
\hline $\mathrm{H}$ & -3.19387198399488 & -2.16579269006684 & -2.98256520596903 \\
\hline $\mathrm{H}$ & -3.62321536099740 & -0.44091078522902 & -2.85512087639287 \\
\hline $\mathrm{C}$ & 1.64945504998139 & 7.55441676902170 & -2.08791426848831 \\
\hline $\mathrm{H}$ & 2.64596123735040 & 7.99293034235665 & -2.22254465857510 \\
\hline $\mathrm{C}$ & -2.07263814789939 & 5.59813237102197 & -1.55714957062894 \\
\hline $\mathrm{C}$ & -1.75487968384529 & -2.36342099076138 & 1.70182170718541 \\
\hline $\mathrm{H}$ & -2.70251792535757 & -1.80092184994339 & 1.60815113478818 \\
\hline $\mathrm{H}$ & -1.81730587034578 & -3.06039626044000 & 2.56164700532427 \\
\hline $\mathrm{H}$ & -1.60634091640395 & -2.93551932389341 & 0.76644049299318 \\
\hline $\mathrm{C}$ & 2.55975726942913 & -0.99379663131267 & -0.55325178729214 \\
\hline $\mathrm{C}$ & -1.96135219600330 & -1.06037065786663 & -1.59005856752152 \\
\hline $\mathrm{C}$ & 2.76241378282584 & 1.02761660931481 & 0.82790125103101 \\
\hline
\end{tabular}




\begin{tabular}{|c|c|c|c|}
\hline $\mathrm{H}$ & 3.78121620687481 & 0.67002061186624 & 1.07631968858672 \\
\hline $\mathrm{H}$ & 2.25082748092301 & 1.23720835294996 & 1.78607177321622 \\
\hline $\mathrm{C}$ & 1.47709548948225 & 6.43507975102691 & -1.25738682416065 \\
\hline $\mathrm{C}$ & 2.72020366991323 & 3.64356453033581 & 0.83642121313929 \\
\hline $\mathrm{H}$ & 2.21658006935421 & 3.41165293348601 & 1.79366787534562 \\
\hline $\mathrm{H}$ & 3.72676345436373 & 4.03311744119199 & 1.08667783134409 \\
\hline $\mathrm{C}$ & 2.81965916063904 & 2.33962028991481 & -0.00460463136591 \\
\hline $\mathrm{H}$ & 1.95894434856808 & 2.32818522940137 & -0.69443698486622 \\
\hline $\mathrm{H}$ & 3.72917558303525 & 2.35671029415822 & -0.63931577207386 \\
\hline $\mathrm{C}$ & -0.73275444312979 & 7.51905650870091 & -2.62716821126282 \\
\hline $\mathrm{H}$ & -1.58632884508401 & 7.93150665414737 & -3.17917717803446 \\
\hline $\mathrm{C}$ & -3.39547089799889 & 5.82266009610262 & -2.23797775339415 \\
\hline $\mathrm{H}$ & -3.71328688457179 & 4.93713332897050 & -2.82895331260870 \\
\hline $\mathrm{H}$ & -3.34244580849037 & 6.67679364322843 & -2.93677615899859 \\
\hline $\mathrm{H}$ & -4.21089402459404 & 6.03823505197946 & -1.51352371422395 \\
\hline $\mathrm{C}$ & -2.53107084928615 & 2.25948656485108 & -1.22076800582965 \\
\hline $\mathrm{H}$ & -1.45615253017461 & 2.27893 & -1.46678169869126 \\
\hline $\mathrm{H}$ & -3.07373884221791 & 2.25572361272514 & -2.18817559653551 \\
\hline $\mathrm{C}$ & 2.45062789299551 & 5.66695605568914 & -0.53057095966360 \\
\hline $\mathrm{C}$ & -0.90327048939605 & 7794 & -1.797 \\
\hline $\mathrm{C}$ & 1.82239017130879 & -2.89202409711106 & -2.12865479957382 \\
\hline $\mathrm{H}$ & 2.83275927891406 & -3.29722338236199 & -2.26446658759746 \\
\hline $\mathrm{C}$ & -0.78691485109905 & -0.33392130289507 & 3.47348406519151 \\
\hline $\mathrm{H}$ & 0.03956432326761 & 0.34985134072819 & 3.74528887045874 \\
\hline $\mathrm{H}$ & -0.95558268234480 & -1.05778887700771 & 4.29716031351530 \\
\hline $\mathrm{H}$ & -1.69845791026716 & 0.27608856237101 & 3.32609786736316 \\
\hline $\mathrm{C}$ & -2.84758142853195 & 3.55891875742118 & -0.42739876125561 \\
\hline $\mathrm{H}$ & -3.87476320559613 & 3.91449140183716 & -0.64226510861889 \\
\hline $\mathrm{H}$ & -2.80385017847699 & 3.33481984959354 & 0.65490714383626 \\
\hline $\mathrm{C}$ & -0.55835644638912 & -2.92780076175869 & -2.67402402163641 \\
\hline $\mathrm{H}$ & -1.39706375068485 & -3.36232257607398 & -3.23183511287534 \\
\hline $\mathrm{C}$ & 4.04667162769103 & -1.22040949622968 & -0.58923217728919 \\
\hline $\mathrm{H}$ & 4.59210412571532 & -0.34346648622817 & -0.99934371411061 \\
\hline $\mathrm{H}$ & 4.29953855237952 & -2.08752453636934 & -1.22541135677598 \\
\hline $\mathrm{H}$ & 4.46546642549378 & -1.41874841326596 & 0.42131919321347 \\
\hline $\mathrm{C}$ & 0.54180504126810 & 8.10134719797342 & -2.76284152609735 \\
\hline $\mathrm{C}$ & 0.73443622927389 & -3.46789547049377 & -2.81150825840436 \\
\hline $\mathrm{C}$ & -1.88692868745249 & 6.89562666151983 & 1.74152149273465 \\
\hline $\mathrm{H}$ & -1.75229331296124 & 7.47814828903177 & 0.81049464623535 \\
\hline $\mathrm{H}$ & -1.96477858157980 & 7.58452989705097 & 2.60658807642965 \\
\hline $\mathrm{H}$ & -2.82165205877490 & 6.31253232838160 & 1.64417994781165 \\
\hline $\mathrm{C}$ & 0.89060396428296 & 6.85525158534020 & 2.41312373878995 \\
\hline $\mathrm{H}$ & 1.74813123493749 & 6.24631182620101 & 2.75532452304361 \\
\hline $\mathrm{H}$ & 0.58287516040722 & 7.55121097446836 & 3.21953494236735 \\
\hline
\end{tabular}




$\begin{array}{llcc}\mathrm{H} & 1.21581254993740 & 7.43285156270072 & 1.52696426746181 \\ \mathrm{C} & -0.87339192183877 & 4.87570874414645 & 3.49753650636520 \\ \mathrm{H} & -1.77326319127751 & 4.24951866382872 & 3.34633099964995 \\ \mathrm{H} & -1.05558471538578 & 5.58976158259522 & 4.32689448827893 \\ \mathrm{H} & -0.03355253767197 & 4.20613127548001 & 3.76366196736523 \\ \mathrm{H} & 0.67653717524116 & 8.97419438551788 & -3.41491087906524 \\ \mathrm{H} & 0.89855019537375 & -4.33005537467601 & -3.47101365261217\end{array}$




\section{References}

(1) Cui, P.; Wang, Q.; McCollom, S. P.; Manor, B. C.; Carroll, P. J.; Tomson, N. C., Angew. Chem. Int. Ed. 2017, 56 (50), 15979-15983.

(2) Zhang, S.; Wang, Q.; Thierer, L. M.; Weberg, A. B.; Gau, M. R.; Carroll, P. J.; Tomson, N. C., Inorg. Chem. 2019, 58 (18), 12234-12244.

(3) Wang, Q.; Zhang, S.; Cui, P.; Weberg, A. B.; Thierer, L. M.; Manor, B. C.; Gau, M. R.; Carroll, P. J.; Tomson, N. C., Inorg. Chem. 2019, doi: 10.1021/acs.inorgchem.9b02339.

(4) Schwindt, M. A.; Lejon, T.; Hegedus, L. S., Organometallics 1990, 9 (10), 2814-2819.

(5) Armarego, W. L. F.; Chai, C. L. L., Chapter 4 - Purification of Organic Chemicals. In Purification of Laboratory Chemicals (Sixth Edition), Armarego, W. L. F.; Chai, C. L. L., Eds. Butterworth-Heinemann: Oxford, 2009; pp 88-444.

(6) M. L. Luetkens, A. P. Sattelberger, H. H. Murray, J. D. Basil, J. P. Fackler, R. A. Jones, D. E. Heaton, in Inorg. Synth., Vol. 28 (Ed.: R. J. Angelici), John Wiley \& Sons, Inc., New York, 1990, pp. 305-310.

(7) CrysAlisPro 1.171.40.53: Rigaku Oxford Diffraction, Rigaku Corporation, Oxford, UK. (2019).

(8) Bruker (2012). SAINT v8.34A. Bruker AXS Inc., M., Wisconsin, USA.

(9) SADABS v2016/2: Krause, L.; Herbst-Irmer, R.; Sheldrick, G. M.; Stalke, D., J. Appl. Crystallogr. 2015, 48 (1), 3-10.

(10) SCALE3 ABSPACK v1.0.7: an Oxford Diffraction program; Oxford Diffraction Ltd: Abingdon, UK, 2005.

(11) F. Neese, WIRES: Comput. Mol. Sci. 2012, 2, 73-78.

(12) a) S. Grimme, J. Comput. Chem. 2006, 27, 1787-1799; b) S. Grimme, J. Antony, S. Ehrlich, H. Krieg, J. Chem. Phys. 2010, 132, 154104-1-19; c) S. Grimme, S. Ehrlich, L. Goerigk, J. Comput. Chem. 2011, 32, 1456-1465.

(13) a) A. Schäfer, H. Horn, R. Ahlrichs, J. Chem. Phys. 1992, 97, 2571-2577; b) A. Schäfer, C. Huber, R. Ahlrichs, J. Chem. Phys. 1994, 100, 5829-5835; c) F. Weigend, R. Ahlrichs, Phys. Chem. Chem. Phys. 2005, 7, 3297-3305.

(14) Pettersen, E. F.; Goddard, T. D.; Huang, C. C.; Couch, G. S.; Greenblatt, D. M.; Meng, E. C.; Ferrin, T. E. UCSF Chimera-A visualization system for exploratory research and analysis. $J$. Comput. Chem. 2004, 25, 1605-1612.

(15) Murray, L. J.; Weare, W. W.; Shearer, J.; Mitchell, A. D.; Abboud, K. A., J. Am. Chem. Soc. 2014, 136 (39), 13502-13505.

(16) Higuchi, J.; Kuriyama, S.; Eizawa, A.; Arashiba, K.; Nakajima, K.; Nishibayashi, Y., Dalton Trans. 2018, 47, 1117-1121.

(17) Sunada, Y.; Imaoka, T.; Nagashima, H., Organometallics, 2010, 29 (23), 6157-6160.

(18) Saouma, C. T.; Moore, C. E.; Rheingold, A. L.; Peters, J. C., Inorg. Chem. 2011, 50 (22), $11285-11287$.

(19) Rose, R. P.; Jones, C.; Schulten, C.; Aldridge, S.; Stasch, A., Chem. Eur. J. 2008, 14 (28), 8477-8480.

(20) Field, L. D.; Guest, R. W.; Turner, P., Inorg. Chem. 2010, 49 (19), 9086-9093.

(21) Zhang, F.; Song, H.; Zhuang, X.; Tung, C.; Wang, W., J. Am. Chem. Soc. 2017, 139 (49), 17775-17778. 
(22) Berke, H.; Bankhardt, W.; Huttner, G.; Seyerl, J. v.; Zsolnai, L., Chem. Ber. 1981, 114, 2754-2768.

(23) Takeshita, T.; Sato, K.; Nakajima, Y., Dalton Trans. 2018, 47, 17004-17010.

(24) Kandler, H.; Gauss, C.; Bidell, W.; Rosenberger, S.; Bürgi, T.; Eremenko, I. L.; Veghini, D.; Orama, O.; Burger, P.; Berke, H., Chem. Eur. J. 1995, 1 (8), 541-548.

(25) Betley, T. A.; Peters, J. C., J. Am. Chem. Soc. 2004, 126 (20), 6252-6254.

(26) Gu, N. X.; Oyala, P. H.; Peters, J. C., J. Am. Chem. Soc. 2018, 140 (20), 6374-6382.

(27) Sunada, Y.; Imaoka, T.; Nagashima, H., Organometallics 2013, 32 (7), 2112-2120.

(28) Yu, R. P.; Darmon, J. M.; Hoyt, J.; Margulieux, G.; Turner, Z. R.; Chirik, P. J., ACS Catal. 2012, $2(8), 1760-1764$.

(29) Doyle, L. R.; Hill, P. J.; Wildgoose, G. G.; Ashley, A. E., Dalton Trans. 2016, 45, 7550-7554.

(30) Rudd, P. A.; Liu, S.; Gagliardi, L.; Young, V. G.; Lu, C. C., J. Am. Chem. Soc. 2011, 133 (51), 20724-20727.

(31) Buscagan, T. M.; Oyala, P. H.; Peters, J. C., Angew. Chem. Int. Ed. 2017, 56, 6921.

(32) Chomitz, W. A.; Arnold, J., Chem. Commun. 2007, 4797-4799.

(33) Sekiguchi, Y.; Kuriyama, S.; Eizawa, A.; Arashiba, K.; Nakajima, K.; Nishibayashi, Y., Chem. Commun. 2017, 53, 12040.

(34) Suess, D. L. M.; Peters, J. C., J. Am. Chem. Soc. 2013, 135 (13), 4938-4941.

(35) Geri, J. B.; Shanahan, P. J.; Szymczak, N. K., J. Am. Chem. Soc. 2017, 139 (16), 5952-5956.

(36) McSkimming, A.; Harman, W. H., J. Am. Chem. Soc. 2015, 137 (28), 8940-8943.

(37) Smith, J. M.; Lachicotte, J. R.; Pittard, K. A.; Cundari, T. R.; Rodgers, G. L.; Rodgers, K. R.; Holland, P. L., J. Am. Chem. Soc. 2001, 123 (37), 9222-9223.

(38) Hein, N. M.; Suzuki, T.; Ogawa, T.; Fryzuk, M. L., Dalton Trans. 2016, 45, 14697-14708.

(39) Cummins, D. C.; Yap, G. P. A.; Theopold, K. H., Eur. J. Inorg. Chem. 2016, 15-16, 2349-2356.

(40) Suzuki, T.; Tsutsui, Y.; Ogawa, T.; Inomata, T.; Ozawa, T.; Sakai, Y.; Fryzuk, M. D.; Masuda, H., Inorg. Chem. 2015, 54 (19), 9271-9281.

(41) Hung, Y.-T.; Yap, G. P. A.; Theopold, K. H., Polyhedron 2019, 157, 381-388.

(42) Vidyaratne, I.; Scott, J.; Gambarotta, S.; Budzelaar, P. H. M., Inorg. Chem. 2007, 46 (17), 7040-7049.

(43) Vidyaratne, I.; Gambarotta, S.; Korobkov, I.; Budzelaar, P. H. M., Inorg. Chem. 2005, 44 (5), 1187-1189.

(44) Pun, D.; Lobkovsky, E.; Chirik, P. J., J. Am. Chem. Soc. 2008, 130 (18), 6047-6054.

(45) Grunze, M.; Golze, M.; Hirschwald, W.; Freund, H.-J.; Pulm, H.; Seip, U.; Tsai, N. C.; Ertl, G.; Küppers, J., Phys. Rev. Letters 1984, 53, 850.

(46) Freund, H.-J.; Bartos, B.; Messmer, R. P., Surf. Sci. 1985, 185, 187-202.

(47) Bart, S. C.; Lobkovsky, E.; Bill, E.; Wieghardt, K.; Chirik, P. J.; Inorg. Chem. 2007, 46 (17), 7055-7063.

(48) Scott, J.; Vidyaratne, I.; Korobkov, I.; Gambarotta, S.; Budzelaar, P. H. M., Inorg. Chem. 2008, 47 (3), 896-911.

(49) Bart, S. C.; Lobkovsky, E.; Chirik, P. J., J. Am. Chem. Soc. 2004, 126 (42), 13794-13807.

(50) Darmon, J. M.; Turner, Z. R.; Lobkovsky, E.; Chirik, P. J., Organometallics 2012, 31 (6), 2275-2285.

(51) Archer, A. M.; Bouwkamp, M. W.; Cortez, M.-P.; Lobkovsky, E.; Chirik, P. J., Organometallics 2006, 25 (18), 4269-4278. 
(52) Stieber, S. C. E.; Milsmann, C.; Hoyt, J. M.; Turner, Z. R.; Finkelstein, K. D.; Wieghardt, K.; Debeer, S.; Chirik, P. J., Inorg. Chem. 2012, 51 (6), 3770-3785.

(53) Atienza, C. C. H.; Tondreau, A. M.; Weller, K. J.; Lewis, K. M.; Cruse, R. W.; Nye, S. A.; Boyer, J. L.; Delis, J. G. P.; Chirik, P. J., ACS Catal. 2012, 2 (10), 2169-2172.

(54) Russell, S. K.; Darmon, J. M.; Lobkovsky, E.; Chirik, P. J.; Inorg. Chem. 2010, 49 (6), 2782-2792.

(55) Fernández, I.; Trovitch, R. J.; Lobkovsky, E.; Chirik, P. J., Organometallics 2008, 27 (1), 109-118.

(56) Neate, P. G. N.; Greenhalgh, M. D.; Brennessel, W. W.; Thomas, S. P.; Neidig, M. L., J. Am. Chem. Soc. 2019, 141 (25), 10099-10108.

(57) Tondreau, A. M.; Milsmann, C.; Patrick, A. D.; Hoyt, H. M.; Lobkovsky, E.; Wieghardt, K.; Chirik, P. J., J. Am. Chem. Soc. 2010, 132 (42), 15046-15059.

(58) Betley, T. A.; Peters, J. C., J. Am. Chem. Soc. 2003, 125 (36), 10782-10783.

(59) McWilliams, S. F.; Bunting, P. C.; Kathiresan, V.; Mercado, B. Q.; Hoffman, B. M.; Long, J. R.; Holland, P. L., Chem. Commun., 2018, 54, 13339-13342.

(60) McWilliams, S. F.; Bill, E.; Lukat-Rodgers, G.; Rodgers, K. R.; Mercado, B. Q.; Holland, P. L., J. Am. Chem. Soc. 2018, 140 (27), 8586-8598.

(61) Grubel, K.; Brennessel, W. W.; Mercado, B. Q.; Holland, P. L., J. Am. Chem. Soc. 2014, 136 (48), 16807-16816.

(62) Smith, J. M.; Lachicotte, J. R.; Pittard, K. A.; Cundari, T. R.; Rodgers, G. L.; Rodgers, K. R.; Holland, P. L., J. Am. Chem. Soc. 2001, 123 (37), 9222-9223.

(63) McWilliams, S. F.; Rodgers, K. R.; Lukat-Rodgers, G.; Mercado, B. Q.; Grubel, K.; Holland, P. L., Inorg. Chem. 2016, 55 (6), 2960-2968. 